\title{
Microglial Activation in Traumatic Brain Injury
}

\author{
Cornelius K. Donat, Gregory Scott, Steve M. Gentleman and Magdalena Sastre *
}

Division of Brain Sciences, Department of Medicine, Imperial College London, London, United Kingdom

Microglia have a variety of functions in the brain, including synaptic pruning, CNS repair and mediating the immune response against peripheral infection. Microglia rapidly become activated in response to CNS damage. Depending on the nature of the stimulus, microglia can take a number of activation states, which correspond to altered microglia morphology, gene expression and function. It has been reported that early microglia activation following traumatic brain injury (TBI) may contribute to the restoration of homeostasis in the brain. On the other hand, if they remain chronically activated, such cells display a classically activated phenotype, releasing pro-inflammatory molecules, resulting in further tissue damage and contributing potentially to neurodegeneration. However, new evidence suggests that this classification is over-simplistic and the balance of activation states can vary at different points. In this article, we review the role of microglia in TBI, analyzing their distribution, morphology and functional phenotype over time in animal models and in humans. Animal studies have allowed genetic and pharmacological manipulations of microglia activation, in order to define their role. In addition, we describe investigations on the in vivo imaging of microglia using translocator protein (TSPO) PET and autoradiography, showing that microglial activation can occur in regions far remote from sites of focal injuries, in humans and animal models of TBI. Finally, we outline some novel potential therapeutic approaches

OPEN ACCESS

Edited by: Isidre Ferrer,

University of Barcelona, Spain

Reviewed by:

Kerry O'Banion,

University of Rochester, United States Kathleen A. Maguire-Zeiss,

Georgetown University, United States

${ }^{*}$ Correspondence:

Magdalena Sastre m.sastre@imperial.ac.uk

Received: 18 April 2017

Accepted: 12 June 2017

Published: 28 June 2017

Citation:

Donat CK, Scott G, Gentleman SM and Sastre M (2017) Microglial Activation in Traumatic Brain Injury. Front. Aging Neurosci. 9:208 doi: 10.3389/fnagi.2017.00208 that prime microglia/macrophages toward the beneficial restorative microglial phenotype after TBI.

Keywords: traumatic brain injury, $\mathrm{CCl}$, microglia, neuroinflammation, TSPO, polarization states

\section{INTRODUCTION}

Traumatic brain injury (TBI) is the biggest cause of death and disability in the under 40s in the developed world (Langlois et al., 2006; Hyder et al., 2007), both in the civilian and military context. The overall majority of TBIs is a single event; however, repeated injuries among soldiers and athletes are associated with the development of Chronic Traumatic Encephalopathy (CTE) although the pathology of CTE can also be seen after single TBI and its causation remains uncertain (Omalu et al., 2011; Goldstein et al., 2012). Survivors often suffer from debilitating cognitive, emotional and physical impairments. This is further aggravated by the constant failure of clinical trials (reviewed in Loane and Faden, 2010; Gruenbaum et al., 2016), leaving patients without any treatment.

Brain injury can trigger neurodegeneration, which is a major determinant of longterm outcome. Head injury is a major risk factor for the development of dementia, suggested by the presence of amyloid- $\beta$ (A $\beta$ ) plaques in around $30 \%$ of post-mortem brain tissue from TBI patients (Roberts et al., 1994). Additionally, amyloid plaques have been observed in surgically removed tissue surrounding contusions in brains of survivors of TBI (Ikonomovic et al., 2004; DeKosky et al., 2007). Recently, it has been shown by Positron Emission Tomography (PET) imaging that the distribution of amyloid plaque in TBI 
survivors overlaps with that in patients with Alzheimer's disease (AD) but also involves the cerebellum, an area of the brain not typically involved in AD (Scott et al., 2016). Other pathological features of $\mathrm{AD}$ are also present in $\mathrm{TBI}$, including increased phosphorylated tau and acetylcholine deficiency (Jordan, 2000; Tran et al., 2011; Goldstein et al., 2012; McKee and Daneshvar, 2015; Shin and Dixon, 2015). In particular, repetitive mild TBI leading to CTE is characterized by perivascular tau pathology, which is also irregularly distributed in the depths of cortical sulci. Another $\mathrm{AD}$ susceptibility factor is the Apolipoprotein E isoform 4 (ApoE4), which has been linked with exiguous neurological outcome after TBI (Verghese et al., 2011). This has been associated with alterations in the integrity of the blood brain barrier (BBB) in individuals with ApoE4 genotype (Methia et al., 2001; Nishitsuji et al., 2011).

Additionally, TBI has been linked with Parkinson's disease (Jafari et al., 2013), various psychiatric disorders leading to increased risk of suicide, an overall increase in mortality (McMillan et al., 2011) and peripheral immune suppression (Hazeldine et al., 2015). There are currently no neuroprotective drug treatments available and a major challenge is to understand how brain trauma causes neurodegeneration and other long-term impairments.

A common feature of the neurological pathologies developing as a consequence of TBI is that they initiate and are potentiated by an inflammatory response. Microglial activation occurs early after experimental (Chiu et al., 2016) and human TBI (Ramlackhansingh et al., 2011; Johnson et al., 2013) and can persist for years, detectable both in vivo and post-mortem. Sites of activation often coincide with neuronal degeneration and axonal abnormality (Maxwell et al., 2010; Giunta et al., 2012). It was hypothesized that the inflammatory response to TBI therefore could be associated with the subsequent development of neurodegenerative disorders. However, new evidence indicates that glial activation may have also reparative/restorative effects. Thus, it is critical to investigate what causes this inflammatory response, what are the consequences for neuronal degeneration and survival and whether this can be modified with antiinflammatory therapeutic approaches.

In this review, we analyse the role of microglia in TBI patients and animal models, from imaging studies used to visualize changes in microglia activation in vivo and ex vivo to studies in post-mortem brains of TBI patients and the prospects of therapies targeting microglia activation in TBI.

\section{MICROGLIA ACTIVATION STATES IN TBI}

TBI occurs when brain structure and physiology are disrupted due to an extrinsic biomechanical insult to the cranium, resulting in neuronal, axonal and vascular damage. In response to TBI, the brain orchestrates a complex immunological tissue reaction similar to ischemic reperfusion injury (Werner and Engelhard, 2007). It was suggested that, as macrophages, microglia can migrate to the site of the injury, in order to establish a protective environment mitigating deleterious consequences of the injury (Faden et al., 2016). The acute function of microglia in response to TBI is to eradicate cellular and molecular debris. Microglial removal of damaged cells is a very important step in the restoration of the normal brain environment. Damaged cells release Danger-associated molecular patterns (DAMPs), which can become potent inflammatory stimuli, resulting in further tissue damage (Solito and Sastre, 2012; Zhang et al., 2012). Moreover, activated microglia are also capable of releasing noxious substances such as pro-inflammatory cytokines, reactive oxygen species (ROS), nitrogen species and excitatory neurotransmitters i.e., glutamate, which exacerbate damage (Kreutzberg, 1996). While pro-inflammatory cytokines are directly deleterious, they also stimulate the release of glutamate from microglia in an autocrine/paracrine fashion. Therefore, depending on the released amount, this can lead to direct neurotoxic effects on neurons, synapses and dendrites, through ionotropic glutamate receptors and interfere with the glutamate buffering ability of astrocytes by inhibiting astrocytic glutamate transporters (Takaki et al., 2012).

It was initially hypothesized that there is a temporary transition in function of the inflammatory milieu, which in the latter phases favors a protracted inflammatory profile associated with chronic microglial activation, precipitating neurological manifestations, although this view has been found to be far too simplistic.

As in other CNS injuries, it seems that microglia activation in TBI results in different phenotypes, corresponding to neurotoxic or neuroprotective priming states. Depending on the stage of the disease and the chronicity, microglia are stimulated differentially and this leads to particular activation states, which correspond to altered microglia morphology, gene expression and function. Microglia are activated in-situ by proinflammatory cytokines such as IFN- $\gamma$, IL- $1 \alpha$, IL-6, and TNF$\alpha$ (Ziebell and Morganti-Kossmann, 2010) and become primed. Microglia morphology can switch from a "normal," ramified shape to a hypertrophic, "bushy" morphology. In response to extensive tissue damage or pathogen invasion, microglia can change into an amoeboid morphology, primarily acting in a phagocytic/macrophage fashion and being difficult to differentiate from infiltrating macrophages (Figure 1). In general, these activation states are classified along a spectrum with M1 or "classically activated" at one end and M2 or "alternatively activated" and the opposite end, similar to macrophages. The proinflammatory M1 phenotype favors the production and release of cytokines that can exacerbate neural injury ( $\mathrm{Hu}$ et al., 2015). In contrast, the M2 is associated with the release of neurotrophic factors that promote repair and a phagocytic role. However, in recent years, transcriptomic analysis has revealed that microglia and macrophages display a much broader transcriptional repertoire than $\mathrm{M} 1$ and $\mathrm{M} 2$, depending on the different environmental signals received (Hickman et al., 2013; Xue et al., 2014). Therefore, it is rare to find clear M1 or M2 microglial phenotypes in chronic diseases, since these states are transitory and eventually include mixed activation states. This seems particularly important in TBI as indicated by the many animal studies showing a mixed expression of different markers associated with both M1 and M2 phenotype (Table 1), also described as transitory state (Mtrans) by some authors (Kumar et al., 2016a). Additionally, the inevitable recruitment of bone 

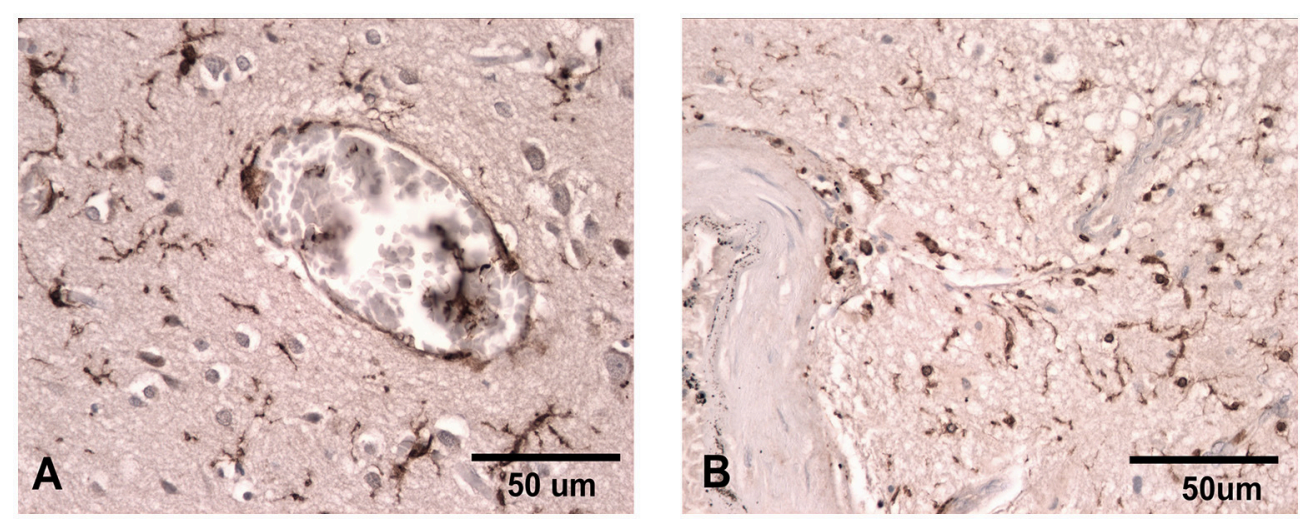

FIGURE 1 | lba-1 staining illustrating the ramified microglial morphology associated with normal surveillance activity (A) compared to that seen in patients where there has been damage to the vasculature and the microglia take on a more rounded phagocytic appearance (B). Scale bar corresponds to $50 \mu \mathrm{M}$.

marrow-derived monocytes into the injured brain parenchyma following experimental TBI and the subsequent differentiation into macrophages complicates the interpretation of the findings, especially with regard to the ability of these cells to change phenotype.

Additional complications arise from the clinical observation that outcome after TBI can be different between male and female adults and post-pubescent adolescents in some but not all studies (Coimbra et al., 2003), with females having lower mortality and less complications (Phelan et al., 2007; Berry et al., 2009; Ley et al., 2013). This can be attributed, at least partially, to astrocytes and microglia, as recently reviewed (Caplan et al., 2017). Gonadal steroid hormones such as progesterone and estradiol are directly involved in the glial response under injury conditions in and during development (Lenz and McCarthy, 2015). Moreover, as progesterone can shift macrophage polarization from M1 to M2 phenotype (Menzies et al., 2011), is seems possible that similar effects could occur in microglia as well, whether the concept of microglia polarization is accepted or not.

The polarization states of microglia could therefore be relevant in the progression of TBI into other neurological disorders such as $\mathrm{AD}$ and could interfere with the recovery of the patients and the effectiveness of particular anti-inflammatory treatment.

In addition, the role of infiltrating macrophages and other immune cells are also relevant in the context of microglia dynamics; injury type and parameters may play an important role, with focal models of TBI generally eliciting a more pronounced and localized inflammatory response due to significant tissue damage and pronounced infiltration of peripheral immune cells through the compromised BBB. In line with this, factors that have been implicated in $\mathrm{BBB}$ breakdown after injury, such as ApoE (mentioned above) and the extracellular enzymes matrix metalloproteinases (MMPs) have been found to contribute to contusion expansion and vasogenic edema after TBI (Guilfoyle et al., 2015).

\section{MICROGLIAL ACTIVATION IN ANIMAL MODELS OF TBI}

In the past five decades, several animal models have been developed to characterize the biomechanical and pathophysiological patterns of TBI and to provide reliably ways to test treatment strategies. These models almost exclusively employ a single-hit injury and only in the past decade repeated injury models emerged that try to mimic the effect of repetitive injuries. The different animal models address distinct pathophysiological aspects of TBI and have different etiological and construct validity (O'Connor et al., 2011). The most widely applied models of TBI are open-head models, that induce an injury via the intact dura mater upon the underlying cortical tissue (reviewed in Xiong et al., 2013), with focal, diffuse and/or mixed injury patterns, depending on the type of injury. In order to do so, rigid impactors (Controlled Cortical Impact, CCI; weight drop, WD), fluidic pressure (Fluid Percussion Injury, FPI) and blast waves are employed. Additionally, high-velocity probes or projectiles are used to model penetrating brain injury. The CCI, FPI and the various WD models have a long history and were extensively characterized in the past, including assessment of long-term outcome (Kochanek et al., 2002; Immonen et al., 2009).

With the recognition that multiple head injuries, even of subthreshold severity, are connected to chronic sequel, such as CTE (McKee et al., 2016; Vile and Atkinson, 2017), new models were developed to account for those findings. A growing number of studies in repeated injury rodent models (Shitaka et al., 2011; Klemenhagen et al., 2013; Mannix et al., 2014; Semple et al., 2016; Robinson et al., 2017) indicate that the glial response, in particular increases in microglia numbers and changes in morphology, shares an overall similar pattern with the traditional single-injury models. Interestingly, a few studies showed that repeated injuries can exacerbate the glial response, e.g., microglia or astrocyte cell density, as compared to a single injury (Ojo et al., 2013; Petraglia et al., 2014; Xu et al., 2016; Gao et al., 2017; Tyburski et al., 2017). Recent findings in human post-mortem 
tissue support the overall notion that repeated injuries result in a stronger glial response by showing that repeated head injury was a predictor of CD68 cell density and phosphorylated tau (Cherry et al., 2016).

The involvement of microglia in the complex pathophysiological cascade following brain injury has long been recognized. Shortly after the description of microglia by Del Rio-Hortega, accumulation of "microglia-like" cells were reported in the tissue around autologous blood injections and a cortical stab wound (Carmichael, 1929; Dunning and Stevenson, 1934) in the rabbit. Similar to reactive astrocytes, microglia are versatile cells, which is exemplified by their heterogeneous morphology (Boche et al., 2013). Perivascular microglia respond to pressure on the thinned mouse skull (Roth et al., 2014) by changing into jellyfish morphology; CCI causes some microglia to align with axon initial segment, the site where action potentials are generated (Baalman et al., 2015) and midline FPI yields several rod-like microglia (Ziebell et al., 2012).

Depending on the employed markers and methods, significantly increasing microglia activation in animal models of TBI is usually apparent from day 1 to 3 post-injury (p.i.) (Bye et al., 2007; Elliott et al., 2011) and can persist chronically beyond 28 days p.i., a usual cut-off time-point for many animal studies. A few studies have elegantly emphasized the longterm nature of microglia activation after experimental TBI, primarily with immunohistochemical analysis showing that major histocompatibility complex (MHC)-II positive cells were still present in the ipsilateral hemisphere at 3 months after WD injury (Holmin and Mathiesen, 1999). Recently, a significant loss of ramified, but increase of hypertrophic microglia in the injured rodent cortex at 1 year after moderate CCI in mice was demonstrated (Loane et al., 2014a). This suggests that, although microglial activation occurs early after TBI, it can change the phenotype and function over time (see Table 1).

\section{Manipulation of Microglial Activation in Animal Models of TBI}

Several studies have investigated the effects of microglial elimination in brain injury models, in order to understand their function and the potential as therapeutic approach. The use of the transgenic CD11b-HSVTK mice in response to ganciclovir (GCV) treatment allowed the study of the depletion of proliferating microglia in models of brain or peripheral nerve injury. These transgenic mice express a mutant form of the gene herpes simplex virus 1 thymidine kinase (HSV-1 TKmt-30) driven by the myeloid specific promoter CD11b. HSV-1 TK is capable of phosphorylating specific nucleoside analogs such as GCV, which lead to inhibition of DNA synthesis and cell death during cell proliferation (Cheng et al., 1983; Faulds and Heel, 1990; Black et al., 1996).

Unexpectedly, eliminating proliferative CD11b positive cells and therefore microglia and potentially macrophages in a model of hypoglossal nerve axotomy did not result in pronounced changes in motor neuron loss (Gowing et al., 2006). In another study, employing a repeated closed-head injury in the CD11b-TK mice, low dose of GCV reduced the microglial population after
TBI but did not alter the extent of axonal injury as visualized by silver staining. Additionally, higher doses were found to be toxic and aggravated the TBI induced damage (Bennett and Brody, 2014).

Rapid elimination of $\sim 95 \%$ of all microglia can be achieved by administration of the colony stimulating factor 1 receptor (CSF1R) inhibitor PLX3397. This approach was used in a model of neuronal injury (Rice et al., 2015) consisting of a diphtheria toxin-induced neuronal lesion in transgenic mice carrying a transgene for the diphtheria toxin A-chain. The diphtheria toxin was activated in transgenic mice by feeding them food without doxycycline, triggering neurotoxicity. The treatment with PLX3397 had different outcomes depending on whether microglia elimination took place in the recovery period only or was administered during the lesion as well as the recovery period. Elimination of microglia after the lesion resulted in improved functional recovery, while microglia depletion during the insult led to greater neuronal loss (Rice et al., 2015). Therefore, in models of CNS damage, it seems that chronically activated microglia display a pro-inflammatory phenotype that may contribute to further synaptic loss. In addition, using the same approach of treatment with the CSF1R inhibitor, it was recently shown that microglia are involved in changes in neuronal network activity and spreading depolarization after excitotoxic lesions and ischemia. Depletion of microglia led to dysregulated neuronal calcium responses, calcium overload and increased neuronal death and infarct size (Szalay et al., 2016), with similar reports in a mouse model of transient focal cerebral ischemia and reperfusion (Jin et al., 2017).

From these findings, it seems obvious that general ablation of microglia is not a productive strategy and perhaps it would be more effective to suppress a specific phenotype at a particular time-point. One approach would be to characterize the different microglial functions based on morphology and phenotype markers in order to find the right time for a microglia-targeting therapeutic strategy.

\section{Polarization of Microglia in TBI Models}

The polarization of microglia is a relatively new concept, not seen without controversy (Ransohoff, 2016), which has been already investigated in a number of studies, primarily in the CCI model in mice. Only a few studies employed rats or other established TBI models, such as FPI and WD. Data from clinically relevant repeated and penetrating models is lacking even for rodents. To the knowledge of the authors, no study has investigated microglia polarization in gyrencephalic models such as ferrets, sheep or pigs, even though these species had previously been employed in CCI, FPI, penetrating, and blast models.

In the established rodent models (see Table 1), data indicates that microglia and infiltrating macrophages, responding to injury, do not lean toward an extreme end of the spectrum, as shown in several studies through the expression of both M1 and M2-like markers in the "acute" phase. However, it seems that in the subsequent "subacute" and "chronic" phase, the expression of M2-like markers is reduced, while M1-like markers are still expressed, indicating that the pro-inflammatory action of microglia/macrophages exacerbates the pathology. In 
TABLE 1 | Microglia/macrophage polarization in experimental models of Traumatic Brain Injury.

\begin{tabular}{|c|c|c|c|c|c|}
\hline References & $\begin{array}{l}\text { Species, strain, } \\
\text { sex }\end{array}$ & $\begin{array}{l}\text { Model, severity and } \\
\text { injury details }\end{array}$ & Time post injury & Key markers & $\begin{array}{l}\text { Simplified key results of } \\
\text { M1/M2-like marker analysis }\end{array}$ \\
\hline Jin et al., 2012 & $\begin{array}{l}\text { Mouse } \\
\text { C57B6/J } \\
\sigma^{2}\end{array}$ & $\begin{array}{l}\mathrm{CCl}, \text { moderate } \\
3 \mathrm{~mm}\end{array}$ & $\begin{array}{l}\text { dpi: } 1,3,714 \text {, } \\
\text { and } 28\end{array}$ & $\begin{array}{l}\text { M1: CD86 } \\
\text { M2: CD206 }\end{array}$ & $\begin{array}{l}\text { M1: } \Delta 7+28 \text { dpi } \\
\text { M2: } \Delta 1 \mathrm{dpi} \text {, Sham levels } 28 \mathrm{dpi}\end{array}$ \\
\hline Bedi et al., 2013 & $\begin{array}{l}\text { Mouse } \\
\text { C57B6 } \\
0^{7}\end{array}$ & $\begin{array}{l}\mathrm{CCl} \text {, moderate } \\
\mathrm{ns}\end{array}$ & $\begin{array}{l}\text { dpi: } \\
1\end{array}$ & $\begin{array}{l}\text { M1: } F_{c} \gamma R \| I / I I I(C D 16 a / b) \\
\text { M2: CD206 }\end{array}$ & M1/2 ratio: $\mathbf{\Delta}$ at $24 \mathrm{hpi}$ \\
\hline Walker et al., 2012 & $\begin{array}{l}\text { Mouse } \\
\text { C57B6 } \\
0^{\pi}\end{array}$ & $\begin{array}{l}\mathrm{CCl} \text {, moderate } \\
\mathrm{ns}\end{array}$ & $\begin{array}{l}\text { hpi: } \\
24,48,72,120\end{array}$ & M2/M1 ratio of CD206/CD86 & M1: $\Delta 24-72$ hpi \\
\hline Hsieh et al., 2013 & $\begin{array}{l}\text { Mouse } \\
\text { C57BL/6 } \\
0^{7}, \\
\text { YARG/Yet40 } \\
\text { knock-in }\end{array}$ & $\begin{array}{l}\mathrm{CCl}, \text { moderate } \\
2 \mathrm{~mm} \text { flat }\end{array}$ & $\begin{array}{l}\text { dpi: } \\
1,4,7,14\end{array}$ & $\begin{array}{l}\text { M1: IL-12p40 } \\
\text { M2: Arg-1 }\end{array}$ & $\begin{array}{l}\text { M1: } \Delta \text { macrophages } 1-14 \text { dpi } \\
\text { M2: } \Delta \text { macrophages } 1 \mathrm{dpi} \\
\text { Mixed M1/M2 expression }\end{array}$ \\
\hline $\begin{array}{l}\text { Tchantchou and Zhang, } \\
2013\end{array}$ & $\begin{array}{l}\text { Mouse } \\
\text { C57B6 } \\
0^{\pi}\end{array}$ & $\begin{array}{l}\mathrm{CCl} \text {, moderate } \\
3 \mathrm{~mm} \text { flat }\end{array}$ & $\begin{array}{l}\text { dpi: } \\
3,7,21\end{array}$ & $\begin{array}{l}\text { M1: iNOS } \\
\text { M2: Arg-1 }\end{array}$ & $\begin{array}{l}\text { M1: } \triangle 3+7 \mathrm{dpi} \text {, } \\
\text { M2: no Arg-1 }\end{array}$ \\
\hline Wang et al., 2013 & $\begin{array}{l}\text { Mouse } \\
\text { C57B6 } \\
0^{7}\end{array}$ & $\begin{array}{l}\mathrm{CCl}, \text { moderate } \\
3 \mathrm{~mm} \text { flat }\end{array}$ & $\begin{array}{l}\text { dpi: } \\
1,3,5,7,14\end{array}$ & $\begin{array}{l}\text { M1: CD16/32, iNOS, CD11b, } \\
\text { CD86 } \\
\text { M2: CD206, IL-10, Ym1/2, } \\
\text { TGF- } \beta \text {, } \\
\text { Arg-1, CCL22 }\end{array}$ & $\begin{array}{l}\text { M1: } \ 5-14 \mathrm{dpi} \\
\text { M2: } \triangle 3-5 \mathrm{dpi}\end{array}$ \\
\hline Wang et al., 2015 & $\begin{array}{l}\text { Mouse } \\
\text { C57B6 } \\
0^{\pi}\end{array}$ & $\begin{array}{l}\mathrm{CCl} \text {, moderate } \\
3 \mathrm{~mm} \text { flat }\end{array}$ & $\begin{array}{l}\text { dpi: } \\
7,35\end{array}$ & $\begin{array}{l}\text { M1: CD16, iNOS } \\
\text { M2: CD206, IL-10 }\end{array}$ & $\begin{array}{l}\text { M1: } \triangle 7 \mathrm{dpi} \\
\text { M2: } \triangle 7 \mathrm{dpi}\end{array}$ \\
\hline Loane et al., 2014a & $\begin{array}{l}\text { Mouse } \\
\text { C57BI/6 } \\
\sigma^{7}\end{array}$ & $\begin{array}{l}\mathrm{CCl} \text {, severe } \\
3.5 \mathrm{~mm} \text { flat }\end{array}$ & $\begin{array}{l}\text { wpi: } \\
1,5,12,52\end{array}$ & $\begin{array}{l}\text { M1: MHC ॥ } \\
\text { M2: Ym-1 }\end{array}$ & $\begin{array}{l}\text { M1/MHC II: } \Delta 1-52 \text { wpi } \\
\text { M2: \ 1-5 wpi, absent 12-54 } \\
\text { wpi }\end{array}$ \\
\hline Loane et al., 2014b & $\begin{array}{l}\text { Mouse } \\
\text { C57BI/6 } \\
\sigma^{7}\end{array}$ & $\begin{array}{l}\mathrm{CCl} \text {, severe } \\
3.5 \mathrm{~mm} \text { flat }\end{array}$ & $\begin{array}{l}\text { dpi: } \\
28\end{array}$ & $\begin{array}{l}\text { M1: iNOS } \\
\text { M2: Arg-1 }\end{array}$ & $\begin{array}{l}\text { M1: } \mathbf{i N O S} / C D 11 b \\
\text { M2: } \mathbf{A r g}-1 / C D 11 b\end{array}$ \\
\hline Kumar et al., 2016a & $\begin{array}{l}\text { Mouse } \\
\text { C57BI/6 } \\
\sigma^{7}\end{array}$ & $\begin{array}{l}\mathrm{CCl} \text {, severe } \\
3.5 \mathrm{~mm} \text { flat }\end{array}$ & $\begin{array}{l}\text { hpi: } \\
1,6,24 \text { dpi: } \\
7\end{array}$ & $\begin{array}{l}\text { mRNA: } \\
\text { M1: IL-1 } \beta, \text { IL-12, TNF- } \alpha \text {, IL-6, } \\
\text { iNOS } \\
\text { M2a: Arg-1, Ym1, CD206, } \\
\text { Fizz-1, } \\
\text { IL-1rn } \\
\text { M2c: IL-4R } \alpha, \text { SOCS3, TGF- } \beta \\
\text { Flow cytometry: } \\
\text { M1: iNOS, IL-12 } \\
\text { M2: TGF- } \beta \text {, } \\
\text { Ym1, CD206, Arg-1 } \\
\text { Histology: } \\
\text { M1: CD16/32, iNOS } \\
\text { Mtrans: CD16/32+TGF- } \beta \text {, } \\
\text { iNOS+, Arg-1 } \\
\text { M2: TGF- } \beta, \text { Arg-1 }\end{array}$ & $\begin{array}{l}\text { Mixed expression of M1/M2 } \\
\text { mRNA and protein } \\
\text { M1/trans dominate contusion } \\
\text { and peri-contusional tissue at } 7 \\
\text { dpi }\end{array}$ \\
\hline
\end{tabular}


TABLE 1 | Continued

\begin{tabular}{|c|c|c|c|c|c|}
\hline References & $\begin{array}{l}\text { Species, strain, } \\
\text { sex }\end{array}$ & $\begin{array}{l}\text { Model, severity and } \\
\text { injury details }\end{array}$ & Time post injury & Key markers & $\begin{array}{l}\text { Simplified key results of } \\
\text { M1/M2-like marker analysis }\end{array}$ \\
\hline Kumar et al., 2016b & $\begin{array}{l}\text { Mouse } \\
\text { C57BI/6 } \\
0^{7} \\
\text { Nox2 -/- }\end{array}$ & $\begin{array}{l}\mathrm{CCl} \text {, severe } \\
3.5 \mathrm{~mm} \text { flat }\end{array}$ & $\begin{array}{l}\text { dpi: } \\
1,3,5,7,21,28\end{array}$ & $\begin{array}{l}\text { M1: IL-1 } 1 \beta, \text { NOS2, TNF- } \alpha \text {, IL-6, } \\
\text { IL-12b } \\
\text { M2: Arg-1, Ym1, SOCS3, Fizz-1, } \\
\text { IL1rn, IL-4R } \alpha \\
\text { M2c: IL-4R } \alpha, \text { SOCS3, TGF- } \beta\end{array}$ & $\begin{array}{l}\text { Mixed expression of M1/2 up to } \\
7 \mathrm{dpi}\end{array}$ \\
\hline Zanier et al., 2014 & $\begin{array}{l}\text { Mouse } \\
\text { C57Bl/6 } \\
\text { ఠ }\end{array}$ & $\begin{array}{l}\mathrm{CCl}, \text { moderate } \\
3 \mathrm{~mm}\end{array}$ & dpi: 3, 7 & $\begin{array}{l}\text { M1: CD11b, TNF- } \alpha, \text { CD68, } \\
\text { CD86, IL-1 } \beta \\
\text { M2a/c: Ym1, Arg-1, CD206, } \\
\text { SOCS3, IL-10 }\end{array}$ & $\begin{array}{l}\text { Mixed expression of } \mathrm{M} 1 \text { and } \mathrm{M} 2 \\
\text { marker mRNA } \\
\text { M1: } \Delta \text { at } 3+7 \text { dpi } \\
\text { M2: } \Delta \text { Ym1, Arg-1, SOCS3 at } 3 \\
\text { dpi }\end{array}$ \\
\hline Desai et al., 2016 & $\begin{array}{l}\text { Mouse } \\
\text { C57BL6/N } \\
\sigma^{7}\end{array}$ & $\begin{array}{l}\mathrm{CCl} \text {, moderate } \\
3 \mathrm{~mm} \text { flat }\end{array}$ & $\begin{array}{l}\text { hpi: } 4 \\
\text { dpi: } \\
1,4\end{array}$ & $\begin{array}{l}\text { M1: CD16, CD32 } \\
\text { M2: CD-206, Arg-1, Ym1 }\end{array}$ & $\begin{array}{l}\text { M1: } \Delta \mathrm{CD} 16, \mathrm{CD} 32 \text { at } 1-4 \text { dpi } \\
\text { M2: } \mathbf{\Delta} \text { CD206, Arg-1, Ym1 at } \\
\text { 1-4 dpi }\end{array}$ \\
\hline Braun et al., 2017 & $\begin{array}{l}\text { Mouse } \\
\text { C57BL/6 } \\
\text { CD-1 } \\
\text { C3H/OuJ } \\
\text { C3H/HeJ } \\
\text { CX3CR1 } \\
\sigma^{7}\end{array}$ & $\begin{array}{l}\text { CCl, severe } \\
3 \mathrm{~mm} \text { convex }\end{array}$ & $\begin{array}{l}\text { hpi: } \\
24,72 \\
\text { wpi: } 3\end{array}$ & $\begin{array}{l}\text { M1: TNF- } \alpha, \text { IL-12 } \\
\text { M2: TGF- } \beta, \text { IL-10 }\end{array}$ & $\begin{array}{l}\text { M1/M2 ratio: } \Delta 24+72 \text { hpi, } 3 \\
\text { wpi }\end{array}$ \\
\hline
\end{tabular}


TABLE 1 | Continued

\begin{tabular}{|c|c|c|c|c|c|}
\hline References & $\begin{array}{l}\text { Species, strain, } \\
\text { sex }\end{array}$ & $\begin{array}{l}\text { Model, severity and } \\
\text { injury details }\end{array}$ & Time post injury & Key markers & $\begin{array}{l}\text { Simplified key results of } \\
\text { M1/M2-like marker analysis }\end{array}$ \\
\hline Ansari, 2015 & $\begin{array}{l}\text { Rat } \\
\text { Sprague-Dawley } \\
\sigma^{7}\end{array}$ & $\begin{array}{l}\mathrm{CCl} \text {, severe } \\
5 \mathrm{~mm} \text { flat }\end{array}$ & $\begin{array}{l}\text { hpi: } \\
2,4,6,10,24\end{array}$ & $\begin{array}{l}\text { M1: TNF- } \alpha, I L-1 \beta, I L-6, I F N-\gamma \\
\text { M2: IL-4, IL-10, IL-13, Arg-1, } \\
\text { Ym1, Fizz1, MRC-1 }\end{array}$ & $\begin{array}{l}\text { Mixed response } \\
\text { M1: } \Delta \text { TNF- } \alpha \text {, IL-1 } \beta \text {, IL-6 } \\
\text { mRNA/protein at 2-10 hpi } \\
\text { M2: } \Delta \text { Arg-1: } 6-24 \text { hpi; Ym1: } 6 \text { hpi, } \\
\text { Fizz1: } 4-24 \text { hpi }\end{array}$ \\
\hline Turtzo et al., 2014 & $\begin{array}{l}\text { Rat } \\
\text { Wistar } \\
\text { q }\end{array}$ & $\begin{array}{l}\mathrm{CCl} \text {, severe } \\
5 \mathrm{~mm} \text { flat }\end{array}$ & $\begin{array}{l}\text { dpi: } \\
\text { 1, 3, 5, 7, 14, } 30 \\
\text { wpi: } \\
8\end{array}$ & $\begin{array}{l}\text { M1: CD40, CD68, TNF- } \alpha, \text { CD86, } \\
\text { CD80, NOS2 } \\
\text { M2: CD163, CD206 }\end{array}$ & $\begin{array}{l}\text { Mixed expression of M1 and M2 } \\
\text { protein expression and mRNA } \\
\text { M1: } \Delta \text { CD86 at 5, } 7 \text { dpi, TNF- } \alpha \text { 1, } 3 \\
\text { dpi } \\
\text { M2: } \triangle \text { CD163 at } 5,7 \text { dpi, CD206 at } 7 \\
\text { dpi }\end{array}$ \\
\hline Barrett et al., 2017 & $\begin{array}{l}\text { Mouse } \\
\text { C57BI/6 } \\
\sigma^{\pi} \\
\text { NOX2 -/- }\end{array}$ & $\begin{array}{l}\mathrm{CCl} \text {, severe } \\
3.5 \mathrm{~mm} \text { flat }\end{array}$ & $\begin{array}{l}\text { dpi: } \\
72\end{array}$ & $\begin{array}{l}\text { M2: IL-4R } \alpha, \text { SOCS3, TGF- } \beta \text {, } \\
\text { SHIP1, Arg-1, Ym1, IL-10 }\end{array}$ & $\begin{array}{l}\text { NOX2 -/- mice show a robust } \\
\text { increase in M2 markers } \\
\Delta \mathrm{IL}-4 \mathrm{R} \alpha, \mathrm{SOCS} 3, \mathrm{TGF}-\beta, \mathrm{SHIP} 1 \text {, } \\
\text { Arg- } 1, \mathrm{Ym} 1, \mathrm{IL}-10 \text { at } 72 \text { hpi vs WT }\end{array}$ \\
\hline Cao et al., 2012 & $\begin{array}{l}\text { Rat } \\
\text { Sprague-Dawley } \\
\sigma^{T}\end{array}$ & $\begin{array}{l}\text { mFPI, moderate } \\
2 \mathrm{~atm} \\
4.8 \mathrm{~mm} \text { craniotomy }\end{array}$ & $\begin{array}{l}\text { dpi: } \\
7,28\end{array}$ & $\begin{array}{l}\text { M1: TNF- } \alpha, \text { CD45 } \\
\text { M2a: Arg-1 } \\
\text { M2c: TGF- } \beta \text { I, TGF- } \beta \text { RII }\end{array}$ & $\begin{array}{l}\text { M1 and M2c marker expression } \\
\text { Cortex: } \\
\text { M1 and M2c: } \Delta \text { nonsignificant CD } 45 \text {, } \\
\text { TGF- } \beta \text { I, TGF- } \beta \text { RII at } 7 \text { dpi, no change } \\
\text { in M2a (Arg- } 1 \text { ) } \\
\text { Thalamus: } \\
\text { M1 and M2C: } \pi \text { CD } 45 \text {, TGF- } \beta \text { I, } \\
\text { TGF- } \beta \text { RII at } 7 \text { and } 28 \text { dpi, no change } \\
\text { in M2a (Arg- } 1 \text { ) }\end{array}$ \\
\hline Bachstetter et al., 2013 & $\begin{array}{l}\text { Mouse } \\
\text { p38 } \alpha \text { MAPK } \\
-/- \\
\text { C57BL/6J } \\
\sigma^{7} / q\end{array}$ & $\begin{array}{l}\mathrm{mFPI}, \text { moderate } \\
1.2 \mathrm{~atm} \\
3 \mathrm{~mm} \text { craniotomy }\end{array}$ & $\begin{array}{l}\text { hpi: } \\
3,9,24 \\
\text { dpi } \\
7\end{array}$ & $\begin{array}{l}\text { M1: IL-1 } \beta, \text { IL-6, TNF- } \alpha, \text { CCL2, } \\
\text { CCL5, CXCL1, CD45, CD68, } \\
\text { MHC } \\
\text { M2: Arg-1, Ym1 }\end{array}$ & $\begin{array}{l}\text { Mixed expression of M1 and M2 } \\
\text { markers mRNA and } \\
\text { cytokines/chemokines } \\
\text { M1: } \mathbf{\Delta} \text { IL-6, TNF- } \alpha, \text { CCL3 3, } 9 \text { hpi, } \\
\text { elevated at } 7 \text { dpi } \\
\text { M2: } \mathbf{\Delta} \text { Arg-1, Ym1 } 9 \text { hpi, } \mathbf{\nabla} \text { below } \\
\text { sham levels at } 7 \text { dpi, Rod-like } \\
\text { microglia } \\
\text { p38 } \text { MAPK -/-: } \\
\mathbf{\Delta} \text { M1 and M2 response } \\
\text { (mRNA/protein) at } 6 \text { hpi } \\
\mathbf{\Delta} \text { motor performance } \\
\nabla \text { Synaptic loss, microglia activation }\end{array}$ \\
\hline Fenn et al., 2014 & $\begin{array}{l}\text { Mouse } \\
\text { BALB/c } \\
0^{\pi}\end{array}$ & $\begin{array}{l}\mathrm{mFPI} \text {, moderate } \\
1.2-1.5 \mathrm{~atm} \\
3 \mathrm{~mm} \text { craniotomy }\end{array}$ & $\begin{array}{l}\text { hpi: } \\
4,72\end{array}$ & $\begin{array}{l}\text { M1: IL-1 } \beta, \text { CD14, TNF- } \alpha \text {, iNOS, } \\
\text { IFN- } \gamma \text {, CCL2 } \\
\text { M2: Arg-1, IL-4R } \alpha, \text { IGF-1, IL-10, } \\
\text { IL4 }\end{array}$ & 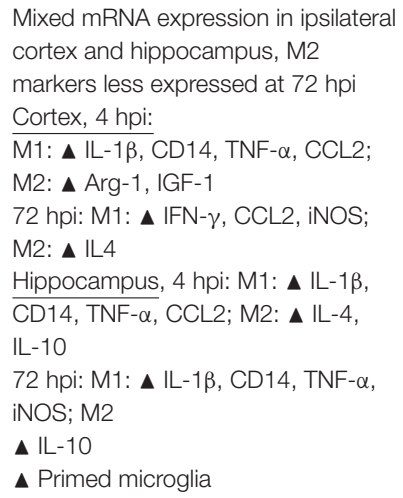 \\
\hline Fenn et al., 2015 & $\begin{array}{l}\text { Mouse } \\
\text { BALB/C } \\
0^{\pi}\end{array}$ & $\begin{array}{l}\mathrm{mFPI}, \text { moderate } \\
1.2-1.5 \mathrm{~atm} \\
3 \mathrm{~mm} \text { craniotomy }\end{array}$ & dpi: 1 & $\begin{array}{l}\text { M1: IL-1 } \beta, \text { CD14, TNF- } \alpha \text {, iNOS } \\
\text { M2: Arg-1, IL-10 }\end{array}$ & $\begin{array}{l}\text { Mixed expression at } 1 \mathrm{dpi} \\
\text { Hippocampus: } \\
\text { M1: } \Delta \mathrm{IL}-1 \beta, \mathrm{CD} 14, \mathrm{TNF}-\alpha, \mathrm{CCL} 2 \\
\text { M2: } \mathbf{A r g}-1\end{array}$ \\
\hline
\end{tabular}


TABLE 1 | Continued

\begin{tabular}{|c|c|c|c|c|c|}
\hline References & $\begin{array}{l}\text { Species, strain, } \\
\text { sex }\end{array}$ & $\begin{array}{l}\text { Model, severity and } \\
\text { injury details }\end{array}$ & Time post injury & Key markers & $\begin{array}{l}\text { Simplified key results of } \\
\text { M1/M2-like marker analysis }\end{array}$ \\
\hline & & & & & $\begin{array}{l}\text { Whole brain CD11b positive cells: } \\
\text { M1: } \Delta \text { IL-1 } \beta, \text { CD14, TNF- } \alpha \\
\text { CCL2, iNOS; } \\
\text { M2: } \Delta \text { Arg-1 }\end{array}$ \\
\hline $\begin{array}{l}\text { Truettner et al., } \\
2016\end{array}$ & $\begin{array}{l}\text { Rat } \\
\text { Sprague-Dawley } \\
\sigma^{\pi}\end{array}$ & $\begin{array}{l}\mathrm{mFPl}, \text { moderate } \\
1.8-2.2 \text { atm } \\
4.8 \mathrm{~mm} \text { craniotomy }\end{array}$ & $\begin{array}{l}\text { hpi: } \\
4,24\end{array}$ & $\begin{array}{l}\text { M1: iNOS, IL-1 } \beta, \text { IL-1 } \alpha, \text { TNF- } \alpha \text {, } \\
\text { IL-6. IL-12, CCL2 } \\
\text { M2: Arg-1, CD163, CD206, } \\
\text { IL-10, } \\
\text { Ym1, TGF- } \beta \\
\text { M1/M2: iNOS/Arg-1 ratio }\end{array}$ & $\begin{array}{l}\text { Mixed expression of M1 and M2 } \\
\text { mRNA at } 4 \text { and } 24 \text { hpi, M1 more } \\
\text { pronounced } \\
\Delta \text { M1 ratio at } 4 \text { h, similar M1/M2 } \\
\text { ratio at } 24 \mathrm{~h} \text { in microglia } \\
\text { mRNA } 4 \text { h: } \\
\text { M1: } \Delta \text { all; } \text { M2: } \mathbf{\Delta} \text { (lower relative } \\
\text { expression) } \\
\text { mRNA } 24 \text { h: } \\
\text { M1: } \Delta \text { TNF- } \alpha \boldsymbol{\Delta} \text { CD206, TGF- } \beta\end{array}$ \\
\hline $\begin{array}{l}\text { Chhor et al., } \\
2016\end{array}$ & $\begin{array}{l}\text { Mouse } \\
\text { OF-1 } \\
\text { Postnatal day } 7 \\
\text { ত/ }\end{array}$ & $\begin{array}{l}\text { WD, closed } \\
2 \mathrm{~mm} \text { footplate } \\
10 \mathrm{~g} \text { weight dropped } \\
10 \mathrm{~cm}\end{array}$ & $\begin{array}{l}\text { hpi: } \\
2,6,14,24 \\
\text { dpi: } \\
5\end{array}$ & $\begin{array}{l}\text { M1 (Cytotoxic): } \\
\text { IL-1 } \alpha, \text { IL-1 } \beta, \text { IL-3, IL-6, IL-9, } \\
\text { IL-12p40, IL-12p70, IL-17, } \\
\text { INF- } \gamma \text {, } \\
\text { TNF- } \alpha, \text { CXCL1, CCL3, CCL4, } \\
\text { CCL5, Cox-2, iNOS, CD32, } \\
\text { CD86 } \\
\text { M2a (Reparative/regenerative): } \\
\text { IL4, IL-13, G-CSF, CCL2, Arg-1, } \\
\text { CD206, IGF-1, Gal-3 } \\
\text { M2c (Immunomodulatory): IL-5, } \\
\text { IL-10, SphK1, IL-1rn, SOCS3, } \\
\text { IL-4ra }\end{array}$ & $\begin{array}{l}\text { Moderate mixed increase in M1 } \\
\text { and M2a/c mRNA } \\
\text { Ipsilateral hemisphere: } \\
\text { M1: } \boldsymbol{\Delta} \text { all markers except TNF- } \alpha \\
\text { and IL-12(p70) between } 6,14 \\
\text { and } 24 \text { hpi } \\
\text { M2a/c: } \boldsymbol{\Delta} \text { all markers except } \\
\text { TNF- } \alpha \text { and IL- } \\
\text { 12(p70) between } 6,14 \text { and } 24 \text { hpi } \\
\text { CD11b+ isolated microglia/macrophages: } \\
\text { M1: } \mathbf{A} \text { CD32/CD86 up to } 1 \text { dpi } \\
\text { M2a/c: } \Delta \text { Arg-1, CD206, } \\
\text { SOCS3, IL-1rn, Gal3 up to } 1 \text { dpi, } \\
\text { IGF-1 at } 5 \text { dpi } \\
\text { Pronounced contralateral effects }\end{array}$ \\
\hline $\begin{array}{l}\text { Semple et al., } \\
2010\end{array}$ & $\begin{array}{l}\text { Mouse } \\
\text { CCL2 -/- } \\
\text { C57BI/6 } \\
\sigma^{7}\end{array}$ & $\begin{array}{l}\text { WD, closed } 333 \mathrm{~g} \\
\text { weight dropped } 2 \\
\mathrm{~cm} \text {, silicone tip }\end{array}$ & $\begin{array}{l}\text { hpi: } 2,4,12,24 \\
\text { dpi: 4, 7, 14, } 28\end{array}$ & $\begin{array}{l}\text { M1: IL-1 } \alpha, I L-1 \beta, I L-6, I L-12 p 40 \\
\text { IL-12p70, CCL3, CXCL1, } \\
\text { CXCL2, CCL5, TNF- } \alpha, \text { INF- } \gamma \\
\text { M2a: G-CSF, IL-10 }\end{array}$ & 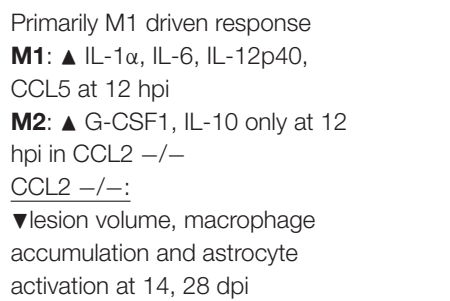 \\
\hline
\end{tabular}

Levels of severity for CCl: Mild: $0.5 \mathrm{~mm}$, Moderate: $0.95-1.5 \mathrm{~mm}$; Severe: $>2.0 \mathrm{~mm}$. $\mathrm{CCl}$, Controlled Cortical Impact; $\mathrm{mFPl}$, midline Fluid Percussion Injury, WD, Weight drop. Dpi, Days post injury; hpi, hours post injury; wpi, weeks post injury.

contrast, studies in non-human primates suggest a trophic role for chronically activated microglia after TBI, indicating a restorative phenotype in the chronic phase (Nagamoto-Combs et al., 2007, 2010).

Another issue is the apparent differences in the expression of murine and human markers of potential microglia polarization, which have been found for macrophages (reviewed in Murray and Wynn, 2011). However, the employed cellular markers of M1-like and the different M2-like microglia/macrophages are not always clearly indicative of the presumed phenotype, which calls for new and more specific markers in murine models (Jablonski et al., 2015). Additionally, mRNA extraction of brain homogenates does not always yield reliable information on the M1/2-like spectrum. Ideally, microglia and macrophages are to be separated by flow cytometry and then assayed for their activation spectrum using transcriptomic analysis (Hickman et al., 2013; Xue et al., 2014).

The studies that employed different therapeutic strategies show that an increase in M2-like markers is often associated with a better cognitive and histopathological outcome (Table 2). It seems therefore imperative for future studies targeting microglia polarization as therapeutic strategies, to assess several markers in the target cells within a sufficient temporal window in order to show a long-term positive outcome.

\section{IMAGING OF MICROGLIA ACTIVATION IN TBI}

Measurement of microglial activation in vivo has become possible using PET and Single Photon Emission Computed 
TABLE 2 | Treatments in experimental models of TBI targeting inflammation and microglia.

\begin{tabular}{|c|c|c|c|c|}
\hline References & Species, Strain, sex & Treatment & Injury & Simplified treatment outcomes \\
\hline Thal et al., 2011 & $\begin{array}{l}\text { Mouse } \\
\text { C57BL6/CrIN } \\
\sigma^{7}\end{array}$ & $\begin{array}{l}\text { Pioglitazone, } \\
\text { rosiglitazone, PPAR } \gamma \\
\text { agonists }\end{array}$ & $\mathrm{CCl}$ & $\begin{array}{l}\text { Pioglitazone: } \\
\nabla \text { Lesion volume at } 24 \mathrm{hpi} \\
\text { M1 } \nabla: \text { iNOS, TNF- } \alpha, \text { IL-1 } 1 \beta \text { mRNA at } \\
24 \text { hpi No beneficial effect of } \\
\text { rosiglitazone }\end{array}$ \\
\hline Bye et al., 2007 & $\begin{array}{l}\text { Mouse } \\
\text { C57BL6 } \\
\text { ఠ }\end{array}$ & Minocycline & Closed-head WD & $\begin{array}{l}4 \text { hpi: } \\
\nabla \text { IL-1 } \beta \\
1 \text { dpi: } \\
\nabla \text { Lesion volume } \\
\nabla \text { Activated microglia accumulation } \\
(\text { F } 4 / 80) \\
\Delta \text { Motor performance } \\
4 \text { dpi: } \\
\text { Beneficial effects on lesion size and } \\
\text { motor function loss } \\
\text { No effect on apoptotic cell death, } \\
\text { neutrophil infiltration }\end{array}$ \\
\hline Homsi et al., 2010 & $\begin{array}{l}\text { Mouse } \\
\text { Swiss } \\
0^{7}\end{array}$ & Minocycline & Closed-head WD & $\begin{array}{l}1 \text { dpi: } \\
\nabla \text { Lesion volume } \\
\nabla \text { Microglia/macrophages (CD11b) } \\
2 \text { dpi -12 wpi: } \\
\nabla \text { Hyper-locomotor activity } \\
\Delta \text { Body weight }\end{array}$ \\
\hline Hanlon et al., 2017 & $\begin{array}{l}\text { Rat } \\
\text { PD } 11 \text { Sprague-Dawley } \\
\sigma^{\pi / q}\end{array}$ & Minocycline & Closed-head CCl & $\begin{array}{l}\text { Short-term treatment ( } 3 \text { days): } \\
\nabla \text { Microglia reactivity in cortex, } \\
\text { hippocampus and white matter at } 3 \\
\text { but not } 7 \text { dpi } \\
\text { dpeurodegeneration at } 3 \text { but not } 7 \\
\text { Long-term treatment ( } 9 \text { days): } \\
\mathbf{A} \text { Microglial reactivity and } \\
\text { neurodegeneration up to } 15 \text { dpi in } \\
\text { cortex and hippocampus } \\
\nabla \text { Spatial memory vs vehicle } \\
\text { No effects of sex }\end{array}$ \\
\hline
\end{tabular}


TABLE 2 | Continued

\begin{tabular}{|c|c|c|c|c|}
\hline References & Species, Strain, sex & Treatment & Injury & Simplified treatment outcomes \\
\hline Chhor et al., 2016 & $\begin{array}{l}\text { Mouse } \\
\text { OF-1 } \\
\text { Postnatal day } 7 \\
\sigma^{\top} / 9\end{array}$ & Minocycline & Closed-head WD & $\begin{array}{l}24 \text { hpi: } \\
\text { M1: } \nabla \text { IL-1 } \beta, \text { IL-6 } 6 \\
\text { M2a/c: } \Delta \text { IGF-1, IL-1rn } \\
\nabla \text { Ventricular volume } \\
\nabla \text { Cleaved caspase } 3 \text { positive cells in } \\
\text { cortex, hippocampus and striatum } \\
\nabla \text { Iba1 positive cells in cortex but not } \\
\text { hippocampus/striatum } \\
\text { No beneficial effects at } 5 \text { dpi }\end{array}$ \\
\hline Lloyd et al., 2008 & $\begin{array}{l}\text { Mouse } \\
\text { CD-1 } \\
\sigma^{7}\end{array}$ & Minozac & Closed-head CCl & $\begin{array}{l}\frac{12 \text { hpi: }}{\text { M1 } \mathbf{V}} \text { : cytokines levels, IL-1 } \beta, I L-6 \text {, } \\
\text { TNF- } \alpha, \text { CCL2 in hippocampus and } \\
\text { cortex } \\
28 \text { dpi: } \\
\nabla \text { Astrogliosis } \\
\nabla \text { Exploratory deficits }\end{array}$ \\
\hline Loane et al., 2014b & $\begin{array}{l}\text { Mouse } \\
\text { C57Bl/6 } \\
\sigma^{7}\end{array}$ & $\begin{array}{l}\text { VU0360172, mGlu5 } \\
\text { positive } \\
\text { allosteric modulator }\end{array}$ & $\mathrm{CCl}$ & $\begin{array}{l}\text { M1: } \nabla \text { iNOS/CD11b } \\
\text { M2: } \Delta \text { Arg-1/CD11b } \\
\Delta \text { motor performance } \\
\nabla \text { lesion size, neurodegeneration }\end{array}$ \\
\hline Wang et al., 2015 & $\begin{array}{l}\text { Mouse } \\
\text { C57B6 } \\
\sigma^{7}\end{array}$ & $\begin{array}{l}\text { Scriptaid, class I/II histone } \\
\text { deacetylase inhibitor }\end{array}$ & $\mathrm{CCl}$ & $\begin{array}{l}\text { M1: } \nabla \text { CD16 cells } \\
\nabla \text { CD16+iNOS mRNA } \\
\text { M2: } \Delta \text { CD206 cells } \Delta \text { CD206 mRNA } \\
\Delta \text { Sensorimotor performance } \\
\Delta \text { White matter integrity }\end{array}$ \\
\hline Kumar et al., 2016a,b & $\begin{array}{l}\text { Mouse } \\
\mathrm{C} 57 \mathrm{BI} / 6 \\
\mathrm{NOX}-1- \\
\sigma^{7}\end{array}$ & $\begin{array}{l}\text { gp91ds-tat, } \\
\text { selective NOX2 inhibitor }\end{array}$ & $\mathrm{CCl}$ & $\begin{array}{l}\text { M1: } \nabla \text { CD16/32 } \\
\text { M2: } \Delta \text { TGF- } \beta \\
\text { M1: } \nabla \text { Nox2 } \\
\text { M2: } \Delta \text { Ym1, Arg-1 } \\
\nabla \text { lesion size, neurodegeneration } \\
\Delta \text { motor/spatial memory } \\
\text { performance }\end{array}$ \\
\hline Menzel et al., 2017 & $\begin{array}{l}\text { Mouse } \\
\text { Progranulin }-/- \text { and WT } \\
\sigma^{\pi}\end{array}$ & $\begin{array}{l}\text { Recombinant progranulin, } \\
\text { pre-injury }\end{array}$ & $\mathrm{CCl}$ & $\begin{array}{l}\nabla \text { M1 cytokines mRNA at } 5 \text { dpi in } \\
\text { progranulin }-/- \text { mice } \\
\nabla \text { Neurological severity score } \\
\nabla \text { Lesion size and white matter } \\
\text { damage } \\
\nabla \text { Astrogliosis }\end{array}$ \\
\hline
\end{tabular}


TABLE 2 | Continued

\begin{tabular}{|c|c|c|c|c|}
\hline References & Species, Strain, sex & Treatment & Injury & Simplified treatment outcomes \\
\hline Cao et al., 2012 & $\begin{array}{l}\text { Rat } \\
\text { Sprague-Dawley } \\
\sigma^{2}\end{array}$ & Ibuprofen & FPI & $\begin{array}{l}\boldsymbol{\nabla} \text { TGF- } \beta \text { I } \\
\text { nonsignificant } \boldsymbol{\nabla} \text { in TSPO, CD45 at } 7 \\
\text { dpi in the thalamus }\end{array}$ \\
\hline $\begin{array}{l}\text { Chio et al., 2013; Cheong } \\
\text { et al., } 2013\end{array}$ & $\begin{array}{l}\text { Rat } \\
\text { Sprague-Dawley } \\
\sigma^{\pi}\end{array}$ & $\begin{array}{l}\text { Etanercept (TNF- } \alpha \\
\text { antagonist) }\end{array}$ & FPI & $\begin{array}{l}\text { 3/7 dpi: } \\
\nabla \text { Neurological severity score } \\
\nabla \text { Iba1/TNF- } \alpha \text { positive cells in } \\
\text { multiple brain regions } \\
\nabla \text { TNF- } \alpha \text { protein levels } \\
\nabla \text { Oedema } \\
\Delta \text { Neurogenesis }\end{array}$ \\
\hline
\end{tabular}

CCl, Controlled Cortical Impact; FPl, Fluid percussion injury, WD, Weight drop.

Dpi, Days post injury, hpi, hours post injury; wpi, weeks post injury;

Green color indicates beneficial, while red indicates detrimental actions of the treatment.

Tomography (SPECT) due to the development of radioligands that bind to the $18-\mathrm{kDa}$ translocator protein (TSPO). TSPO is a five-transmembrane domain protein localized in the outer mitochondrial membrane (Jaremko et al., 2014). The best studied putative function of TSPO relates to its role in transporting cholesterol into the mitochondrial inner membrane space, the rate-limiting step of steroid and neurosteroid biosynthesis (Papadopoulos et al., 2006). However, this function has been lately questioned due to the findings from knockout mice that did not show abnormal steroidogenesis (Morohaku et al., 2014; Tu et al., 2014). Despite almost 40 years of study, the precise functional role of TSPO is far from clear (Selvaraj and Stocco, 2015).

\section{Imaging of Activated Microglia after Experimental TBI}

Autoradiographic studies and PET using TSPO ligands in experimental TBI have informed our understanding of the timecourse and spatial distribution of microglial activation following brain trauma. The first TSPO ligand, PK11195, was synthesized in the 1980s. Autoradiographic studies localized binding of radiolabelled PK11195 to activated microglia (Banati et al., 1997) and, as a result, $\left[{ }^{11} \mathrm{C}\right] \mathrm{PK} 11195$ was adopted as a PET radioligand to image neuroinflammation in vivo (Liu et al., 2014). Increased TSPO-specific radioligand binding was detected as early as $6 \mathrm{~h}$ after CCI in rats, with significantly elevated radioligand binding in the ipsilateral and contralateral cortices at $24 \mathrm{~h}$ and delayed thalamic upregulation at 28 days p.i. (Donat et al., 2016). Additionally, we previously observed a small increase of $\left[{ }^{3} \mathrm{H}\right] \mathrm{PK} 11195$ binding in several brain regions of newborn piglets subjected to FPI at $6 \mathrm{~h}$ p.i. (Donat et al., 2014). Similarly, increased TSPO up-regulation indicative of microglia activation was reported in models including FPI (Yu et al., 2010; Cao et al., 2012), CCI (Venneti et al., 2007; Folkersma et al., 2011b; Wang et al., 2014), penetrating brain injury (Miyazawa et al., 1995; Grossman et al., 2012), closed-head injury (CHI) (Grossman et al., 2003) and dynamic cortical deformation (Soustiel et al., 2008).

In vivo studies employing $\left[{ }^{11} \mathrm{C}\right] \mathrm{PK} 11195 \mathrm{PET}$ in rats following CCI 1 day after injury showed no differences in whole brain uptake compared to baseline values or sham-treated controls, whereas scans at 10 days showed significantly increased uptake (Folkersma et al., 2011b). In another CCI rat model, uptake of $\left[{ }^{18} \mathrm{~F}\right] \mathrm{DPA}-714$ (a second-generation TSPO ligand) was observed on day 2 after injury, peaking on day 6 , and remaining elevated to day 16 (Wang et al., 2014). Similar findings were seen in a weightdrop mouse model, no differences were observed in $\left[{ }^{18} \mathrm{~F}\right] \mathrm{DPA}-$ 714 binding at day 1 , but increased uptake in focal brain lesions on repeated scans at days 7 and 16 (Israel et al., 2016).

Several experimental studies have also shown that TSPO expression increases not only in the vicinity of lesioned areas but also at locations remote from sites of focal damage. Following $\mathrm{CCI}$ in rats, the ipsilateral thalamus and hippocampus show increased $\left[{ }^{3} \mathrm{H}\right] \mathrm{PK} 11195$ binding between 3 and 14 days after injury (Raghavendra Rao et al., 2000). In the FPI model, increased uptake of $\left[{ }^{18} \mathrm{~F}\right]$-fluoroethyl-DAA1106 (a secondgeneration TSPO ligand) appears in the ipsilateral striatum up to 4 weeks' post-injury (Yu et al., 2010). In our own study, using a CCI model in rats, we found increased binding of $\left[{ }^{123} \mathrm{I}\right] \mathrm{CLINDE}$ (a second-generation TSPO ligand) in contralateral motor cortex early after injury $(24 \mathrm{~h})$, whereas increased binding in the ipsilateral thalamus was observed first at 28 days post-injury (Donat et al., 2016).

However, TSPO up-regulation does not appear to relay any information on potential polarization pattern (Kim and $\mathrm{Yu}$, 2015). In a mouse model of intracranial hemorrhage, microglia exclusively expressed TSPO and in these cells CD16/32 (M1like) or CD206 (M2-like) were evenly distributed (Bonsack et al., 2016).

While TSPO seems a more general biomarker of glia activation, likely showing the mitochondrial changes due to the increased metabolic demand resulting from glia activation and cell proliferation (Liu et al., 2014), other targets expressed by microglia (e.g., cannabinoid type 2 receptor, $\mathrm{P} 2 \mathrm{X} 7$ and P2Y12 receptors) could be more indicative of microglial polarization, although less tracers are available for these targets.

\section{Imaging Microglia in Humans}

In humans, $\left[{ }^{11} \mathrm{C}\right] \mathrm{PK} 11195$ PET has demonstrated increased TSPO expression in a variety of neuroinflammatory conditions (Owen and Matthews, 2011), including herpes encephalitis (Cagnin et al., 2001b), Alzheimer's disease (Cagnin et al., 2001a), 


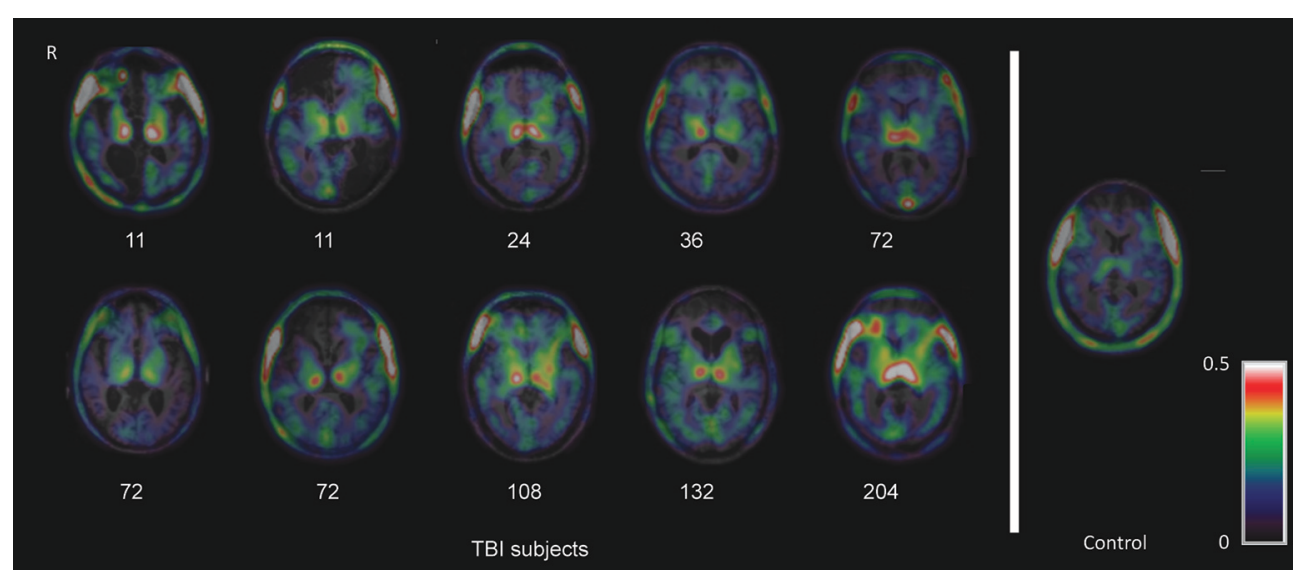

FIGURE 2 | Imaging of chronic microglial activation after TBI. Images of [11C]PK11195 PET images are shown superimposed on the T1 MRI scan at the level of the thalamus for $10 \mathrm{TBI}$ patients, 11 months to 17 years after injury, and a representative control participant. Numbers indicate time since injury (months). $R=$ right. The figure has been reproduced with permission of the copyright holder (Ramlackhansingh et al., 2011).

multiple sclerosis (Banati et al., 2000), and stroke (Gerhard et al., 2005). In the normal human brain, $\left[{ }^{11} \mathrm{C}\right] \mathrm{PK} 11195$ uptake is generally low, but with relatively high binding in subcortical structures, including midbrain, thalamus and putamen (Kumar et al., 2012; Kobayashi et al., 2017). The relatively increased TSPO ligand binding observed in midbrain, thalamus and basal ganglia (Kumar et al., 2012) is in keeping with the high density of microglia found in these regions in the mouse (Lawson et al., 1990) and human brain (Mittelbronn et al., 2001) in comparison to the cerebral cortex. However, increased TSPO ligand binding may reflect an increase in TSPO density rather than the number of cells expressing TSPO. These regions are highly connected through white matter to widespread brain regions. Owing to their dense connectivity, it is possible that even subtle neuronal injury, as may arise in normal aging, may result in the accumulation of secondary microglial activation in these regions, and therefore increased TSPO binding.

PET imaging with TSPO ligands provides a method to visualize microglial activity following TBI in vivo, although the specificity of TSPO for microglia still remains controversial. In an early study, we used $\left[{ }^{11} \mathrm{C}\right] \mathrm{PK} 11195$ PET to investigate a group of 10 patients in the chronic phase 11 months to 17 years after single moderate-severe TBI (Figure 2; Ramlackhansingh et al., 2011). $\left[{ }^{11} \mathrm{C}\right] \mathrm{PK} 11195$ binding potential was significantly increased in subcortical structures, particularly the thalami, putamen, and parts of the white matter. In keeping with animal work, this chronic microglial activation was seen in regions remote from focal damage and was also reduced around areas of focal injury. High $\left[{ }^{11} \mathrm{C}\right] \mathrm{PK} 11195$ binding in the thalamus was associated with more severe cognitive impairment, suggesting either that microglial activation might be directly contributing to cognitive impairment or that this was a response to underlying brain injury that caused the cognitive impairment.

These initial findings have been replicated and extended in further studies. A second study by another group also used $\left[{ }^{11} \mathrm{C}\right] \mathrm{PK} 11195$ PET following TBI (Folkersma et al., 2011a). They again showed increased binding in subcortical regions remote from the focal traumatic pathology, which included the thalamus, putamen, pons, and hippocampus. Increased microglial activation has also been observed in sportsmen exposed to repetitive TBI (Coughlin et al., 2015, 2017). A group of 14 active or recently retired National Football League players with a history of concussions were studied using the second generation TSPO ligand $\left[{ }^{11} \mathrm{C}\right] \mathrm{DPA}-$ 713. Increased binding was seen predominantly in medial temporal lobe regions in sportsmen who also showed subtle evidence of white matter damage on diffusion MRI. In our follow-up study, we used the second generation TSPO ligand $\left[{ }^{11}\right.$ C]PBR28 (Scott et al., 2015). A similar distribution of subcortical microglial activation was again seen years after single moderate/severe TBI. Prominent white matter microglial activation in areas of white matter damage was measured using magnetic resonance imaging (MRI). In addition, areas with high microglial activation showed high levels of brain atrophy over the next 6 months. This suggests that chronically activated microglia are seen in areas of persistent white matter damage, which are progressively degenerating even many years after injury.

These studies consistently show microglial activation in regions far removed from sites of focal injuries. Whilst at first glance surprising, the observation is in keeping with the evolution of microglial activation after experimental TBI (Donat et al., 2016) and is likely to reflect slowly progressive changes within damaged white matter, in particular Wallerian degeneration (Figure 3). In our initial study, the level of thalamic microglial activation after TBI showed a strong correlation with the level of traumatic axonal injury in thalamo-cortical projections. This suggests that traumatic axonal injury might be playing a causative role in increasing chronic microglial activation (Scott et al., 2015). The effect is not specific to TBI and a similar progression of microglial activity is seen following lacunar infarcts. This suggests that as the after effects of axonal injury develop and Wallerian degeneration progresses, microglial activation might be observed in cortical and subcortical regions remote from the initial injury. 


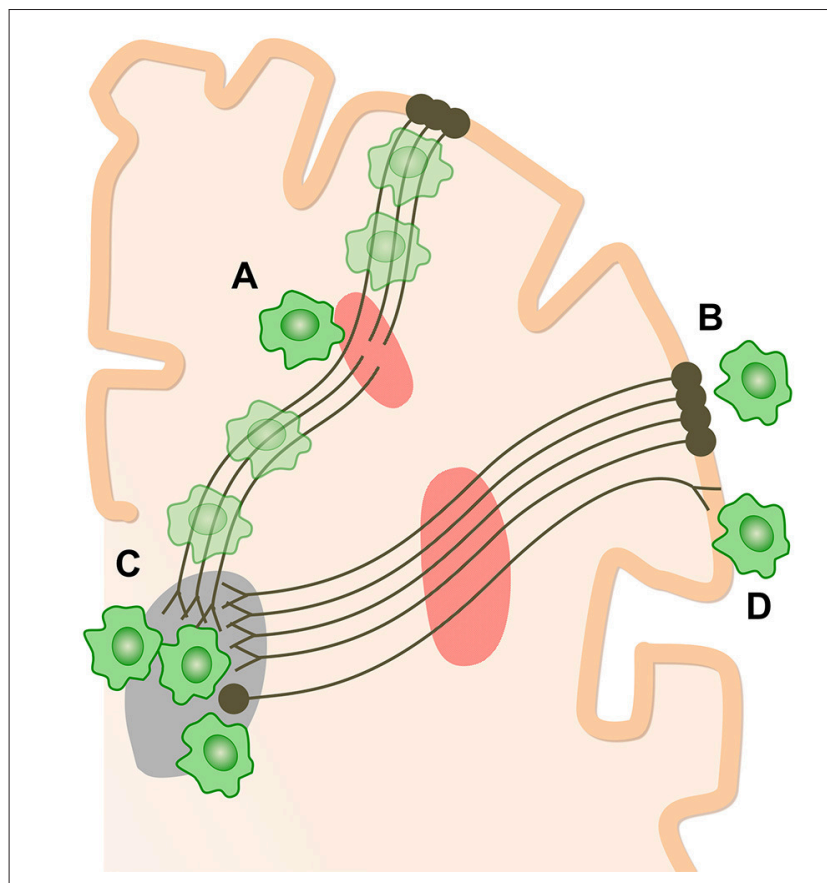

FIGURE 3 | How chronic microglial activation and axonal injury may be linked after TBI. Microglial activation (green cells) and traumatic axonal injury in thalamo-cortical white matter tracts (red areas) have been demonstrated after TBI. Sites of chronic microglial activation can co-localize with axonal abnormality (A) as well as along the entire axonal tract affected by injury. Remote from sites of primary axonal injury, microglia may be observed both in retrograde projection areas, toward the cell bodies of damaged neurons (B), and in anterograde areas (C,D). The thalamus is a highly-connected structure. Thalamic microglial activation may be observed after TBI because of the high density of connections to damaged axons. The number of cortico-thalamic projections far exceeds thalamo-cortical projections. If microglial activation preferentially favors anterograde involvement, then relatively increased activation would be expected in the thalamus $\mathbf{( C )}$ compared to corresponding cortical areas (B)

A number of factors might explain the thalamic rather than cortical preponderance of microglial activation after TBI. Firstly, there is a high density of projection neurons converging in the thalamus, which might lead to a regional amplification of widespread, but sub-threshold cortical pathology (Cagnin et al., 2003). Secondly, as cortico-thalamic projections are 10 times more numerous that thalamo-cortical projections, a more intense microglial reaction to anterograde neurodegeneration might contribute to the persistent microglial reaction in the thalamus (Figure 3).

\section{ANTI-INFLAMMATORY TREATMENTS TARGETING MICROGLIA ACTIVATION IN TBI}

The relevance of microglia in the general pathophysiological response to TBI is recognized as a potential therapeutic avenue (reviewed in Chio et al., 2015) and has prompted several studies to investigate the effects of certain drugs on microglial polarization in brain injury models. Although studies in animal models indicate that microglia/macrophages respond to TBI with a transient M2 phenotype, followed by a shift to M1, and that the number of M1 cells is strongly correlated with the severity of white matter injury (Koh and DiPietro, 2011), this may differ in non-human and human primates. Therefore, therapies that prime microglia/macrophages toward the beneficial M2 phenotype after TBI may offer new anti-inflammatory strategies (Table 2). Several of these drugs, including minocycline, minozac, etanercept and the PPAR agonists fenofibrate and pioglitazone are FDA approved (Garrido-Mesa et al., 2013; Kim et al., 2014) confirming their safety and tolerability.

\section{Progranulin}

In response to $\mathrm{TBI}$, microglia/macrophages and astrocytes release inflammatory mediators with dual effects on secondary brain damage progression. The neurotrophic and antiinflammatory glycoprotein progranulin (PGRN) attenuates neuronal damage and microglia/macrophage activation in brain injury but mechanisms are still elusive. Intracerebroventricular administration of recombinant PGRN in mice immediately before trauma reduced brain damage and neurological deficits, and restored normal levels of cytokine transcription, axonal injury and astrogliosis 5 days after TBI in granulin knockout mice (Menzel et al., 2017).

\section{Minocycline}

Minocycline is a tetracycline derivative with anti-inflammatory and neuroprotective properties. Some of the proposed mechanisms for the anti-inflammatory properties for minocycline include the inhibitory effects on the activities of key enzymes, like iNOS, MMPs and PLA2 (Garrido-Mesa et al., 2013). In particular, it has been shown that it is able to inhibit M1 polarization state of microglia (Kobayashi et al., 2013), through the inhibition of NFאB and the interference with MAPK pathways. Minocycline significantly inhibited retinal neuroinflammation in an ischemia reperfusion (IR) model, characterized by inflammatory gene expression, leukocyte adhesion and invasion, and vascular permeability, however it failed to block neurodegeneration (Abcouwer et al., 2013). The effects of minocycline treatment in an animal model of TBI (closed head CCI in the neonate rat) revealed that the protective effects could be detected short term after injury (3 days after minocycline treatment) but not in chronic treatment (for 9 days after injury), in which microglial reactivity and neurodegeneration in all regions examined were exacerbated in minocycline-treated brain-injured animals (Hanlon et al., 2016, 2017). However, in other TBI models, such as blast injury, minocycline appears to prevent the development of neurobehavioral abnormalities (Kovesdi et al., 2012).

\section{Minozac}

Minozac (Mzc) is an anti-inflammatory molecule that selectively reduces excessive pro-inflammatory cytokine production by activated glia toward basal levels. There is evidence that administration of Mzc in a mouse closed-head, CCI at 3 and $9 \mathrm{~h}$ following TBI attenuates the acute increase in proinflammatory 
cytokine and chemokine levels and reduces the longer-term astrocyte activation, neurologic injury and neurobehavioral deficits observed over a 28 -day recovery period (Lloyd et al., 2008)

\section{Pharmacological Inhibition of TNF- $\alpha$}

Pharmacological inhibition of TNF- $\alpha$ using etanercept has been shown to reduce the expression of microglial TNF- $\alpha$ in rodents subjected to FPI (Cheong et al., 2013; Chio et al., 2013) and improve the neurological outcome after stroke and TBI in humans (Tobinick et al., 2012). Etanercept also seems to be able to stimulate neurogenesis in rats (Cheong et al., 2013).

\section{Modulation of Glutamate Receptors}

Although glutamate released by microglia may be related to a neurotoxic effect, it was shown that activation of metabotropic glutamate receptor 5 (mGluR5) on microglia is a novel mechanism to attenuate M1-like microglial activation and associated microglial-mediated neurotoxicity, suggesting a selfregulatory mechanism. Positive allosteric activation of mGluR5 has powerful neuroprotective effects in experimental models of CNS injury (Loane et al., 2014b).

\section{PPAR Agonists}

In traumatic brain injury, the $\operatorname{PPAR} \alpha$ agonist fenofibrate appears to represent a highly promising new anti-inflammatory compound. Besson et al. (2005) assessed the pharmacological role of fenofibrate in the FPI model in adult male SpragueDawley rats. The study revealed that the administration of fenofibrate during a clinically relevant therapeutic "time window of opportunity" at $1 \mathrm{~h}$ after trauma mediated a significant posttraumatic neuroprotection. This was demonstrated by improved neurological scores in the fenofibrate group at $24 \mathrm{~h}$ and 7 days after trauma, compared to vehicle-treated animals (Besson et al., 2005). In the case of PPAR $\gamma$ agonists, both pioglitazone and rosiglitazone seem to have protective effects, in particular pioglitazone reduced the histological damage and inflammation in the CCI model of TBI (Thal et al., 2011).

\section{HDAC Inhibition}

HDAC inhibitors have been found to have anti-inflammatory and neuroprotective effect in models of TBI. Scriptaid, a novel inhibitor of class I/II HDACs, was found to facilitate and enhance recovery of motor functions after CCI and protected white matter up to 35 day after TBI, as shown by reductions in abnormally dephosphorylated neurofilament protein, increases in myelin basic protein, anatomic preservation of myelinated axons, and improved nerve conduction (Wang et al., 2015). Furthermore, Scriptaid shifted microglia/macrophage polarization toward the protective M2 phenotype and mitigated inflammation. In primary co-cultures of microglia and oligodendrocytes, Scriptaid increased expression of microglial glycogen synthase kinase 3 beta (GSK3 $\beta$ ), which phosphorylated and inactivated phosphatase and tensin homolog (PTEN), thereby enhancing phosphatidylinositide 3-kinases (PI3K)/Akt signaling and polarizing microglia toward M2. The increase in GSK3 $\beta$ in microglia and their phenotypic switch to M2 was associated with increased preservation of neighboring oligodendrocytes.

\section{Nicotinamide Adenine Dinucleotide Phosphate (NADPH) oxidase}

Nicotinamide adenine dinucleotide phosphate (NADPH) oxidase inhibitors were able to alter M1-/M2-like balance in favor of the anti-inflammatory M2-like phenotype in a CCI model of TBI (Kumar et al., 2016a,b).

\section{CONCLUSIONS}

In recent years, there has been a growing interest in investigating the activation of microglia in TBI, because of the potential role in the progression of those patients to neurodegenerative and psychiatric diseases. The studies of microglia in animal models of TBI have allowed the manipulation of microglial numbers (by genetic or pharmacological ablation), indicating their important role in neuroprotection especially at early times post-injury, although this sequence seems to be model and species-specific. In addition, this has provided insights into the time course of the different profile of microglial activation phenotypes in the injury site and the spreading of inflammation to other areas of the brain, such as the thalamus.

In vivo molecular imaging of TSPO potentially provides an extremely useful biomarker of microglial activation and the effect of immunomodulatory drugs. In TBI patients, it has allowed the observation of chronically activated microglia located in areas of persistent white matter damage, even long time post injury. However, it currently only provides a one-dimensional measure, i.e., the amount of microglial activation within a particular brain region. It is clearly too simplistic to describe microglial activation in vivo along a single dimension (i.e., from "low activity" to "high activity"). Crucially, on the basis of current evidence, TSPO molecular imaging cannot discriminate activation phenotype, but probably reflects the (potentially uneven) summation of activity from microglia across all functional states.

In addition, there has been some debate regarding the possibility that certain TSPO ligands also recognize astrocytes or other immune cells in the brains of TBI patients. Other limitations of the application of second-generation ligands include that the binding affinities are influenced by a common polymorphism (rs6971) in the TSPO gene which causes a single amino acid substitution (A147T) in the protein (Owen et al., 2012). Because 147T TSPO binds ligands with lower affinity than $147 \mathrm{~A}$, this produces three classes of binding affinity across a population, which studies must therefore control for. Even after accounting for TSPO genotype, however, many second-generation TSPO ligands show high between-subject variability in uptake when using analysis methods which rely on measurement of the ligand in arterial blood (Guo et al., 2012). As for $\left[{ }^{11} \mathrm{C}\right] \mathrm{PK} 11195$, high and variable plasma protein binding may be a factor (Lockhart et al., 2003). Methods of analysis such as the simplified reference tissue model (SRTM) have been developed that do not require arterial blood data, but rather use the PET 
imaging data from a reference region (or reference tissue) instead (Gunn et al., 1997).

To improve the interpretation of the TSPO signal, a detailed characterization is needed of how TSPO expression in humans varies with the diversity of microglial phenotypes seen in vivo. Future TBI work needs to provide a description of the time-course of microglial phenotype change after TBI and its relationship to TSPO expression. Novel PET ligands showing specificity for distinct functional subtypes of microglial activation would be of great utility, with cannabinoid type 2 and purinergic receptors, such as $\mathrm{P} 2 \mathrm{Y} 12$ and $\mathrm{P} 2 \mathrm{X} 7$, possibly providing suitable targets in the future. Another option is to combine TSPO PET imaging with different biomarkers that disambiguate the TSPO signal in a particular context, in particular neurofilament light (NFL). NFL levels in CSF have been proposed as a valid biomarker to accurately assess the level of trauma and predict the clinical outcome of the patient (Zhang et al., 2016).

Recent investigations have indicated that simple suppression of microglia activation can exert only limited beneficial effect and the inhibition of M1-like responses might be detrimental similar to a simple stimulation of M2-like phenotypes, as indicated by increased fibrosis which seems modulated by Arginase- 1 in peripheral and central infection (Hesse et al., 2001; Aldrich and Kielian, 2011). In addition, pharmacological treatment with the anti-inflammatory drugs tested in several models may not have similar effects when administered in TBI patients, as seen in clinical trials for $\mathrm{AD}$ (Lleo et al., 2007), limiting the

\section{REFERENCES}

Abcouwer, S. F., Lin, C. M., Shanmugam, S., Muthusamy, A., Barber, A. J., and Antonetti, D. A. (2013). Minocycline prevents retinal inflammation and vascular permeability following ischemia-reperfusion injury. $J$. Neuroinflammat. 10:149. doi: 10.1186/1742-2094-10-149

Aldrich, A., and Kielian, T. (2011). Central nervous system fibrosis is associated with fibrocyte-like infiltrates. Am. J. Pathol. 179, 2952-2962. doi: 10.1016/j.ajpath.2011.08.036

Ansari, M. A. (2015). Temporal profile of M1 and M2 responses in the hippocampus following early $24 \mathrm{~h}$ of neurotrauma. J. Neurol. Sci. 357, 41-49. doi: 10.1016/j.jns.2015.06.062

Baalman, K., Marin, M. A., Ho, T. S., Godoy, M., Cherian, L., Robertson, C., et al. (2015). Axon initial segment-associated microglia. J. Neurosci. 35, 2283-2292. doi: 10.1523/JNEUROSCI.3751-14.2015

Bachstetter, A. D., Rowe, R. K., Kaneko, M., Goulding, D., Lifshitz, J., and Van Eldik, L. J. (2013). The p38alpha MAPK regulates microglial responsiveness to diffuse traumatic brain injury. J. Neurosci. 33, 6143-6153. doi: 10.1523/JNEUROSCI.5399-12.2013

Banati, R. B., Myers, R., and Kreutzberg, G. W. (1997). PK ('peripheral benzodiazepine')--binding sites in the CNS indicate early and discrete brain lesions: microautoradiographic detection of [3H]PK11195 binding to activated microglia. J. Neurocytol. 26, 77-82. doi: 10.1023/A:1018567510105

Banati, R. B., Newcombe, J., Gunn, R. N., Cagnin, A., Turkheimer, F., Heppner, F., et al. (2000). The peripheral benzodiazepine binding site in the brain in multiple sclerosis: quantitative in vivo imaging of microglia as a measure of disease activity. Brain 123 (Pt 11), 2321-2337. doi: 10.1093/brain/123.11.2321

Barrett, J. P., Henry, R. J., Villapol, S., Stoica, B. A., Kumar, A., Burns, M. P., et al. (2017). NOX2 deficiency alters macrophage phenotype through an IL10/STAT3 dependent mechanism: implications for traumatic brain injury. J. Neuroinflammat. 14, 65. doi: 10.1186/s12974-017-0843-4 potential therapeutic impact. It seems therefore imperative for future studies that target microglia polarization as therapeutic strategies, to assess several markers in the target cells within a sufficient temporal window in order to show a long-term positive outcome.

\section{AUTHOR CONTRIBUTIONS}

MS wrote most of the introduction, the manipulation of microglia in animal models and the treatments section; CD wrote most of the section on animal models of TBI and preclinical TSPO imaging in animal models, made the tables, the references and wrote part of the conclusions; GS wrote most of the part of the imaging with TSPO, made two figures and part of the conclusions; SG contributed with the image of microglia (Figure 1) and edited the manuscript.

\section{FUNDING}

ISSF Networks of Excellence award 2015 (reference 105603/Z/14/Z, Wellcome Trust).

\section{ACKNOWLEDGMENTS}

We thank Prof. David J. Sharp (Imperial College London) for critical reading of the manuscript.

Bedi, S. S., Smith, P., Hetz, R. A., Xue, H., and Cox, C. S. (2013). Immunomagnetic enrichment and flow cytometric characterization of mouse microglia. J. Neurosci. Methods 219, 176-182. doi: 10.1016/j.jneumeth.2013. 07.017

Bennett, R. E., and Brody, D. L. (2014). Acute reduction of microglia does not alter axonal injury in a mouse model of repetitive concussive traumatic brain injury. J. Neurotrauma. 31, 1647-1663. doi: 10.1089/neu.2013.3320

Berry, C., Ley, E. J., Tillou, A., Cryer, G., Margulies, D. R., and Salim, A. (2009). The effect of gender on patients with moderate to severe head injuries. J. Trauma 67, 950-953. doi: 10.1097/TA.0b013e3181ba3354

Besson, V. C., Chen, X. R., Plotkine, M., and Marchand-Verrecchia, C. (2005). Fenofibrate, a peroxisome proliferator-activated receptor alpha agonist, exerts neuroprotective effects in traumatic brain injury. Neurosci. Lett. 388, 7-12. doi: 10.1016/j.neulet.2005.06.019

Black, M. E., Newcomb, T. G., Wilson, H. M., and Loeb, L. A. (1996). Creation of drug-specific herpes simplex virus type 1 thymidine kinase mutants for gene therapy. Proc. Natl. Acad. Sci. U.S.A. 93, 3525-3529. doi: 10.1073/pnas.93.8.3525

Boche, D., Perry, V. H., and Nicoll, J. A. (2013). Review: activation patterns of microglia and their identification in the human brain. Neuropathol. Appl. Neurobiol. 39, 3-18. doi: 10.1111/nan.12011

Bonsack, F. IV, Alleyne, C. H. Jr., and Sukumari-Ramesh, S. (2016). Augmented expression of TSPO after intracerebral hemorrhage: a role in inflammation? J. Neuroinflammat. 13:151. doi: 10.1186/s12974-016-0619-2

Braun, M., Vaibhav, K., Saad, N., Fatima, S., Brann, D. W., Vender, J. R., et al. (2017). Activation of Myeloid TLR4 Mediates T Lymphocyte polarization after Traumatic Brain Injury. J. Immunol. 198, 3615-3626. doi: 10.4049/jimmunol.1601948

Bye, N., Habgood, M. D., Callaway, J. K., Malakooti, N., Potter, A., Kossmann, T., et al. (2007). Transient neuroprotection by minocycline following traumatic brain injury is associated with attenuated microglial activation but no 
changes in cell apoptosis or neutrophil infiltration. Exp. Neurol. 204, 220-233. doi: 10.1016/j.expneurol.2006.10.013

Cagnin, A., Brooks, D. J., Kennedy, A. M., Gunn, R. N., Myers, R., Turkheimer, F. E., et al. (2001a). In-vivo measurement of activated microglia in dementia. Lancet 358, 461-467. doi: 10.1016/S0140-6736(01)05625-2

Cagnin, A., Gerhard, A., and Banati, R. B. (2003). In vivo Imaging of Neuroinflammation in Neurodegenerative Diseases. (Neuroinflammation: Mechanisms and Management). Totowa, NJ: Humana Press Inc.

Cagnin, A., Myers, R., Gunn, R. N., Lawrence, A. D., Stevens, T., Kreutzberg, G. W., et al. (2001b). In vivo visualization of activated glia by [11C] (R)-PK11195-PET following herpes encephalitis reveals projected neuronal damage beyond the primary focal lesion. Brain 124(Pt 10), 2014-2027. doi: 10.1093/brain/124.10.2014

Cao, T., Thomas, T. C., Ziebell, J. M., Pauly, J. R., and Lifshitz, J. (2012). Morphological and genetic activation of microglia after diffuse traumatic brain injury in the rat. Neuroscience 225, 65-75. doi: 10.1016/j.neuroscience.2012.08.058

Caplan, H. W., Cox, C. S., and Bedi, S. S. (2017). Do microglia play a role in sex differences in TBI? J. Neurosci. Res. 95, 509-517. doi: 10.1002/jnr. 23854

Carmichael, E. A. (1929). Microglia: an experimental study in rabbits after intracerebral injection of blood. J. Neurol. Psychopathol. 9, 209-216. doi: 10.1136/jnnp.s1-9.35.209

Cheng, Y. C., Huang, E. S., Lin, J. C., Mar, E. C., Pagano, J. S., Dutschman, G. E., et al. (1983). Unique spectrum of activity of 9-[(1,3-dihydroxy-2propoxy)methyl]-guanine against herpesviruses in vitro and its mode of action against herpes simplex virus type 1. Proc. Natl. Acad. Sci. U.S.A. 80, 2767-2770. doi: 10.1073/pnas.80.9.2767

Cheong, C. U., Chang, C. P., Chao, C. M., Cheng, B. C., Yang, C. Z., and Chio, C. C. (2013). Etanercept attenuates traumatic brain injury in rats by reducing brain TNF- alpha contents and by stimulating newly formed neurogenesis. Mediat. Inflamm. 2013:620837. doi: 10.1155/2013/620837

Cherry, J. D., Tripodis, Y., Alvarez, V. E., Huber, B., Kiernan, P. T., Daneshvar, D. H., et al. (2016). Microglial neuroinflammation contributes to tau accumulation in chronic traumatic encephalopathy. Acta Neuropathol. Commun. 4, 112. doi: $10.1186 / \mathrm{s} 40478-016-0382-8$

Chhor, V., Moretti, R., Le Charpentier, T., Sigaut, S., Lebon, S., Schwendimann, L., et al. (2016). Role of microglia in a mouse model of paediatric traumatic brain injury. Brain Behav. Immun. 63, 197-209. doi: 10.1016/j.bbi.2016. 11.001

Chio, C. C., Chang, C. H., Wang, C. C., Cheong, C. U., Chao, C. M., Cheng, B. C., et al. (2013). Etanercept attenuates traumatic brain injury in rats by reducing early microglial expression of tumor necrosis factor-alpha. BMC Neurosci. 14:33. doi: $10.1186 / 1471-2202-14-33$

Chio, C. C., Lin, M. T., and Chang, C. P. (2015). Microglial activation as a compelling target for treating acute traumatic brain injury. Curr. Med. Chem. 22, 759-770. doi: 10.2174/0929867321666141106124657

Chiu, C. C., Liao, Y. E., Yang, L. Y., Wang, J. Y., Tweedie, D., Karnati, H. K., et al. (2016). Neuroinflammation in animal models of traumatic brain injury. J. Neurosci. Methods 272, 38-49. doi: 10.1016/j.jneumeth.2016. 06.018

Coimbra, R., Hoyt, D. B., Potenza, B. M., Fortlage, D., and HollingsworthFridlund, P. (2003). Does sexual dimorphism influence outcome of traumatic brain injury patients? The answer is no! J. Trauma. 54, 689-700. doi: 10.1097/01.TA.0000058314.31655.5F

Coughlin, J. M., Wang, Y., Minn, I., Bienko, N., Ambinder, E. B., Xu, X., et al. (2017). Imaging of glial cell activation and white matter integrity in brains of active and recently retired national football league players. JAMA Neurol. 74, 67-74. doi: 10.1001/jamaneurol.2016.3764

Coughlin, J. M., Wang, Y., Munro, C. A., Ma, S., Yue, C., Chen, S., et al. (2015). Neuroinflammation and brain atrophy in former NFL players: an in vivo multimodal imaging pilot study. Neurobiol. Dis. 74, 58-65. doi: 10.1016/j.nbd.2014.10.019

DeKosky, S. T., Abrahamson, E. E., Ciallella, J. R., Paljug, W. R., Wisniewski, S. R., Clark, R. S., et al. (2007). Association of increased cortical soluble abeta 42 levels with diffuse plaques after severe brain injury in humans. Arch. Neurol. 64, 541-544. doi: 10.1001/archneur.64.4.541
Desai, A., Park, T., Barnes, J., Kevala, K., Chen, H., and Kim, H. Y. (2016). Reduced acute neuroinflammation and improved functional recovery after traumatic brain injury by alpha-linolenic acid supplementation in mice. $J$. Neuroinflammat. 13:253. doi: 10.1186/s12974-016-0714-4

Donat, C. K., Fischer, F., Walter, B., Deuther-Conrad, W., Brodhun, M., Bauer, R., et al. (2014). Early increase of cannabinoid receptor density after experimental traumatic brain injury in the newborn piglet. Acta Neurobiol. Exp. (Wars) 74, 197-210.

Donat, C. K., Gaber, K., Meixensberger, J., Brust, P., Pinborg, L. H., Hansen, H. H., et al. (2016). Changes in binding of [(123)I]CLINDE, a high-affinity translocator protein $18 \mathrm{kDa}$ (TSPO) selective radioligand in a rat model of traumatic brain injury. Neuromol. Med. 18, 158-169. doi: 10.1007/s12017-016-8385-y

Dunning, H. S., and Stevenson, L. (1934). Microglia-like cells and their reaction following injury to the liver, spleen and kidney. Am. J. Pathol. 10, 343-348.7

Elliott, M. B., Tuma, R. F., Amenta, P. S., Barbe, M. F., and Jallo, J. I. (2011). Acute effects of a selective cannabinoid-2 receptor agonist on neuroinflammation in a model of traumatic brain injury. J. Neurotrauma 28 , 973-981. doi: 10.1089/neu.2010.1672

Faden, A. I., Wu, J., Stoica, B. A., and Loane, D. J. (2016). Progressive inflammation-mediated neurodegeneration after traumatic brain or spinal cord injury. Br. J. Pharmacol. 173, 681-691. doi: 10.1111/bph.13179

Faulds, D., and Heel, R. C. (1990). Ganciclovir. A review of its antiviral activity, pharmacokinetic properties and therapeutic efficacy in cytomegalovirus infections. Drugs 39, 597-638. doi: 10.2165/00003495-199039040-00008

Febinger, H. Y., Thomasy, H. E., Pavlova, M. N., Ringgold, K. M., Barf, P. R., George, A. M., et al. (2015). Time-dependent effects of CX3CR1 in a mouse model of mild traumatic brain injury. J. Neuroinflammat. 12:154. doi: 10.1186/s12974-015-0386-5

Fenn, A. M., Gensel, J. C., Huang, Y., Popovich, P. G., Lifshitz, J., and Godbout, J. P. (2014). Immune activation promotes depression 1 month after diffuse brain injury: a role for primed microglia. Biol. Psychiatry 76, 575-584. doi: 10.1016/j.biopsych.2013.10.014

Fenn, A. M., Skendelas, J. P., Moussa, D. N., Muccigrosso, M. M., Popovich, P. G., Lifshitz, J., et al. (2015). Methylene blue attenuates traumatic brain injuryassociated neuroinflammation and acute depressive-like behavior in mice. $J$. Neurotrauma. 32, 127-138. doi: 10.1089/neu.2014.3514

Folkersma, H., Boellaard, R., Yaqub, M., Kloet, R. W., Windhorst, A. D., Lammertsma, A. A., et al. (2011a). Widespread and prolonged increase in (R)11C-PK11195 binding after traumatic brain injury. J. Nucl. Med. 52, 1235-1239. doi: $10.2967 /$ jnumed.110.084061

Folkersma, H., Foster Dingley, J. C., van Berckel, B. N., Rozemuller, A., Boellaard, R., Huisman, M. C., et al. (2011b). Increased cerebral (R)-[11C]-PK11195 uptake and glutamate release in a rat model of traumatic brain injury: a longitudinal pilot study. J. Neuroinflammat. 8:67. doi: 10.1186/1742-2094-8-67

Gao, H., Han, Z., Bai, R., Huang, S., Ge, X., Chen, F., et al. (2017). The accumulation of brain injury leads to severe neuropathological and neurobehavioral changes after repetitive mild traumatic brain injury. Brain Res. 1657, 1-8. doi: 10.1016/j.brainres.2016.11.028

Garrido-Mesa, N., Zarzuelo, A., and Galvez, J. (2013). Minocycline: far beyond an antibiotic. Br. J. Pharmacol., 169, 337-352. doi: 10.1111/bph.12139

Gerhard, A., Schwarz, J., Myers, R., Wise, R., and Banati, R. B. (2005). Evolution of microglial activation in patients after ischemic stroke: a [11C](R)-PK11195 PET study. Neuroimage 24, 591-595. doi: 10.1016/j.neuroimage.2004.09.034

Giunta, B., Obregon, D., Velisetty, R., Sanberg, P. R., Borlongan, C. V., and Tan, J. (2012). The immunology of traumatic brain injury: a prime target for Alzheimer's disease prevention. J. Neuroinflammat. 9:185. doi: 10.1186/1742-2094-9-185

Goldstein, L. E., Fisher, A. M., Tagge, C. A., Zhang, X. L., Velisek, L., Sullivan, J. A., et al. (2012). Chronic traumatic encephalopathy in blast-exposed military veterans and a blast neurotrauma mouse model. Sci. Transl. Med. 4, 134ra160. doi: 10.1126/scitranslmed.3003716

Gowing, G., Vallieres, L., and Julien, J. P. (2006). Mouse model for ablation of proliferating microglia in acute CNS injuries. Glia 53, 331-337. doi: $10.1002 /$ glia.20288

Grossman, R., Paden, C. M., Fry, P. A., Rhodes, R. S., and Biegon, A. (2012). Persistent region-dependent neuroinflammation, NMDA receptor loss and 
atrophy in an animal model of penetrating brain injury. Fut. Neurol. 7, 329-339. doi: $10.2217 /$ fnl.12.25

Grossman, R., Shohami, E., Alexandrovich, A., Yatsiv, I., Kloog, Y., and Biegon, A. (2003). Increase in peripheral benzodiazepine receptors and loss of glutamate NMDA receptors in a mouse model of closed head injury: a quantitative autoradiographic study. Neuroimage 20, 1971-1981. doi: 10.1016/j.neuroimage.2003.06.003

Gruenbaum, S. E., Zlotnik, A., Gruenbaum, B. F., Hersey, D., and Bilotta, F. (2016). Pharmacologic neuroprotection for functional outcomes after traumatic Brain injury: a systematic review of the clinical literature. CNS Drugs 30, 791-806. doi: 10.1007/s40263-016-0355-2

Guilfoyle, M. R., Carpenter, K. L., Helmy, A., Pickard, J. D., Menon, D. K., and Hutchinson, P. J. (2015). Matrix metalloproteinase expression in contusional traumatic brain injury: a paired microdialysis study. J. Neurotrauma 32, 1553-1559. doi: 10.1089/neu.2014.3764

Gunn, R. N., Lammertsma, A. A., Hume, S. P., and Cunningham, V. J. (1997). Parametric imaging of ligand-receptor binding in PET using a simplified reference region model. Neuroimage 6, 279-287. doi: 10.1006/nimg. 1997.0303

Guo, Q., Owen, D. R., Rabiner, E. A., Turkheimer, F. E., and Gunn, R. N. (2012). Identifying improved TSPO PET imaging probes through biomathematics: the impact of multiple TSPO binding sites in vivo. Neuroimage 60, 902-910. doi: 10.1016/j.neuroimage.2011.12.078

Hanlon, L. A., Huh, J. W., and Raghupathi, R. (2016). Minocycline transiently reduces microglia/macrophage activation but exacerbates cognitive deficits following repetitive traumatic brain injury in the neonatal rat. J. Neuropathol. Exp. Neurol. 75, 214-226. doi: 10.1093/jnen/nlv021

Hanlon, L. A., Raghupathi, R., and Huh, J. W. (2017). Differential effects of minocycline on microglial activation and neurodegeneration following closed head injury in the neonate rat. Exp. Neurol. 290, 1-14. doi: 10.1016/j.expneurol.2016.12.010

Hazeldine, J., Lord, J. M., and Belli, A. (2015). Traumatic brain injury and peripheral immune suppression: primer and prospectus. Front. Neurol. 6:235. doi: 10.3389/fneur.2015.00235

Hesse, M., Modolell, M., La Flamme, A. C., Schito, M., Fuentes, J. M., Cheever, A. W., et al. (2001). Differential regulation of nitric oxide synthase-2 and arginase- 1 by type 1/type 2 cytokines in vivo: granulomatous pathology is shaped by the pattern of L-arginine metabolism. J. Immunol. 167, 6533-6544. doi: 10.4049/jimmunol.167.11.6533

Hickman, S. E., Kingery, N. D., Ohsumi, T. K., Borowsky, M. L., Wang, L. C., Means, T. K., et al. (2013). The microglial sensome revealed by direct RNA sequencing. Nat. Neurosci. 16, 1896-1905. doi: 10.1038/nn.3554

Holmin, S., and Mathiesen, T. (1999). Long-term intracerebral inflammatory response after experimental focal brain injury in rat. Neuroreport 10, 1889-1891. doi: 10.1097/00001756-199906230-00017

Homsi, S., Piaggio, T., Croci, N., Noble, F., Plotkine, M., Marchand-Leroux, C., et al. (2010). Blockade of acute microglial activation by minocycline promotes neuroprotection and reduces locomotor hyperactivity after closed head injury in mice: a twelve-week follow-up study. J. Neurotrauma 27, 911-921. doi: 10.1089/neu.2009.1223

Hsieh, C. L., Kim, C. C., Ryba, B. E., Niemi, E. C., Bando, J. K., Locksley, R. M., et al. (2013). Traumatic brain injury induces macrophage subsets in the brain. Eur. J. Immunol. 43, 2010-2022. doi: 10.1002/eji.201243084

Hu, X., Leak, R. K., Shi, Y., Suenaga, J., Gao, Y., Zheng, P., et al. (2015). Microglial and macrophage polarization-new prospects for brain repair. Nat. Rev. Neurol. 11, 56-64. doi: 10.1038/nrneurol.2014.207

Hyder, A. A., Wunderlich, C. A., Puvanachandra, P., Gururaj, G., and Kobusingye, O. C. (2007). The impact of traumatic brain injuries: a global perspective. NeuroRehabilitation 22, 341-353.

Ikonomovic, M. D., Uryu, K., Abrahamson, E. E., Ciallella, J. R., Trojanowski, J. Q., Lee, V. M., et al. (2004). Alzheimer's pathology in human temporal cortex surgically excised after severe brain injury. Exp. Neurol. 190, 192-203. doi: 10.1016/j.expneurol.2004.06.011

Immonen, R. J., Kharatishvili, I., Grohn, H., Pitkanen, A., and Grohn, O. H. (2009). Quantitative MRI predicts long-term structural and functional outcome after experimental traumatic brain injury. Neuroimage 45, 1-9. doi: 10.1016/j.neuroimage.2008.11.022
Israel, I., Ohsiek, A., Al-Momani, E., Albert-Weissenberger, C., Stetter, C., Mencl, S., et al. (2016). Combined [(18)F]DPA-714 micro-positron emission tomography and autoradiography imaging of microglia activation after closed head injury in mice. J. Neuroinflammat. 13:140. doi: 10.1186/s12974-016-0604-9

Jablonski, K. A., Amici, S. A., Webb, L. M., Ruiz-Rosado Jde, D., Popovich, P. G., Partida-Sanchez, S., et al. (2015). Novel Markers to Delineate Murine M1 and M2 Macrophages. PLoS ONE 10:e0145342. doi: 10.1371/journal.pone.0145342

Jafari, S., Etminan, M., Aminzadeh, F., and Samii, A. (2013). Head injury and risk of Parkinson disease: a systematic review and meta-analysis. Mov. Disord. 28, 1222-1229. doi: $10.1002 / \mathrm{mds} .25458$

Jaremko, L., Jaremko, M., Giller, K., Becker, S., and Zweckstetter, M. (2014). Structure of the mitochondrial translocator protein in complex with a diagnostic ligand. Science 343, 1363-1366. doi: 10.1126/science.1248725

Jin, W. N., Shi, S. X., Li, Z., Li, M., Wood, K., Gonzales, R. J., et al. (2017). Depletion of microglia exacerbates postischemic inflammation and brain injury. J. Cereb. Blood Flow Metab. 37, 2224-2236. doi: 10.1177/0271678x17694185

Jin, X., Ishii, H., Bai, Z., Itokazu, T., and Yamashita, T. (2012). Temporal changes in cell marker expression and cellular infiltration in a controlled cortical impact model in adult male C57BL/6 mice. PLoS ONE 7:e41892. doi: 10.1371/journal.pone.0041892

Johnson, V. E., Stewart, J. E., Begbie, F. D., Trojanowski, J. Q., Smith, D. H., and Stewart, W. (2013). Inflammation and white matter degeneration persist for years after a single traumatic brain injury. Brain 136(Pt 1), 28-42. doi: 10.1093/brain/aws322

Jordan, B. D. (2000). Chronic traumatic brain injury associated with boxing. Semin Neurol. 20, 179-185. doi: 10.1055/s-2000-9826

Kim, J. H., Jenrow, K. A., and Brown, S. L. (2014). Mechanisms of radiationinduced normal tissue toxicity and implications for future clinical trials. Radiat. Oncol. J. 32, 103-115. doi: 10.3857/roj.2014.32.3.103

Kim, E. J., and Yu, S. W. (2015). Translocator protein 18 kDa (TSPO): old dogma, new mice, new structure, and new questions for neuroprotection. Neural Regen. Res. 10, 878-880. doi: 10.4103/1673-5374.158338

Klemenhagen, K. C., O’Brien, S. P., and Brody, D. L. (2013). Repetitive concussive traumatic brain injury interacts with post-injury foot shock stress to worsen social and depression-like behavior in mice. PLoS ONE 8:e74510. doi: 10.1371/journal.pone.0074510

Kobayashi, K., Imagama, S., Ohgomori, T., Hirano, K., Uchimura, K., Sakamoto, K., et al. (2013). Minocycline selectively inhibits M1 polarization of microglia. Cell Death Dis. 4:e525. doi: 10.1038/cddis.2013.54

Kobayashi, M., Jiang, T., Telu, S., Zoghbi, S. S., Gunn, R. N., Rabiner, E. A., et al. (2017). 11C-DPA-713 has much greater specific binding to translocator protein $18 \mathrm{kDa}$ (TSPO) in human brain than 11C-( R)-PK11195. J. Cereb. Blood Flow Metab. 271678x17699223. doi: 10.1177/0271678x176 99223

Kochanek, P. M., Hendrich, K. S., Dixon, C. E., Schiding, J. K., Williams, D. S., and Ho, C. (2002). Cerebral blood flow at one year after controlled cortical impact in rats: assessment by magnetic resonance imaging. J. Neurotrauma 19, 1029-1037. doi: 10.1089/089771502760341947

Koh, T. J., and DiPietro, L. A. (2011). Inflammation and wound healing: the role of the macrophage. Expert Rev. Mol. Med. 13:e23. doi: $10.1017 / \mathrm{s} 1462399411001943$

Kovesdi, E., Kamnaksh, A., Wingo, D., Ahmed, F., Grunberg, N. E., Long, J. B., et al. (2012). Acute minocycline treatment mitigates the symptoms of mild blast-induced traumatic brain injury. Front. Neurol. 3:111. doi: 10.3389/fneur.2012.00111

Kreutzberg, G. W. (1996). Microglia: a sensor for pathological events in the CNS. Trends Neurosci. 19, 312-318. doi: 10.1016/0166-2236(96)10049-7

Kumar, A., Alvarez-Croda, D. M., Stoica, B. A., Faden, A. I., and Loane, D. J. (2016a). Microglial/Macrophage polarization dynamics following traumatic brain injury. J. Neurotrauma 33, 1732-1750. doi: 10.1089/neu.2015.4268

Kumar, A., Barrett, J. P., Alvarez-Croda, D. M., Stoica, B. A., Faden, A. I., and Loane, D. J. (2016b). NOX2 drives M1-like microglial/macrophage activation and neurodegeneration following experimental traumatic brain injury. Brain Behav. Immun. 58, 291-309. doi: 10.1016/j.bbi.2016.07.158

Kumar, A., Muzik, O., Shandal, V., Chugani, D., Chakraborty, P., and Chugani, H. T. (2012). Evaluation of age-related changes in translocator protein (TSPO) 
in human brain using (11)C-[R]-PK11195 PET. J. Neuroinflammat. 9:232. doi: 10.1186/1742-2094-9-232

Kumar, A., Stoica, B. A., Sabirzhanov, B., Burns, M. P., Faden, A. I., and Loane, D. J. (2013). Traumatic brain injury in aged animals increases lesion size and chronically alters microglial/macrophage classical and alternative activation states. Neurobiol. Aging 34, 1397-1411. doi: 10.1016/j.neurobiolaging.2012.11.013

Langlois, J. A., Rutland-Brown, W., and Wald, M. M. (2006). The epidemiology and impact of traumatic brain injury: a brief overview. J. Head Trauma Rehabil, 21, 375-378. doi: 10.1097/00001199-200609000-00001

Lawson, L. J., Perry, V. H., Dri, P., and Gordon, S. (1990). Heterogeneity in the distribution and morphology of microglia in the normal adult mouse brain. Neuroscience 39, 151-170. doi: 10.1016/0306-4522(90)90229-W

Lenz, K. M., and McCarthy, M. M. (2015). A starring role for microglia in brain sex differences. Neuroscientist 21, 306-321. doi: 10.1177/1073858414536468

Ley, E. J., Short, S. S., Liou, D. Z., Singer, M. B., Mirocha, J., Melo, N., et al. (2013). Gender impacts mortality after traumatic brain injury in teenagers. J. Trauma Acute Care Surg. 75, 682-686. doi: 10.1097/TA.0b013e31829d024f

Liu, G. J., Middleton, R. J., Hatty, C. R., Kam, W. W., Chan, R., Pham, T., et al. (2014). The $18 \mathrm{kDa}$ translocator protein, microglia and neuroinflammation. Brain Pathol. 24, 631-653. doi: 10.1111/bpa.12196

Lleo, A., Galea, E., and Sastre, M. (2007). Molecular targets of non-steroidal anti-inflammatory drugs in neurodegenerative diseases. Cell Mol. Life Sci. 64, 1403-118. doi: 10.1007/s00018-007-6516-1

Lloyd, E., Somera-Molina, K., Van Eldik, L. J., Watterson, D. M., and Wainwright, M. S. (2008). Suppression of acute proinflammatory cytokine and chemokine upregulation by post-injury administration of a novel small molecule improves long-term neurologic outcome in a mouse model of traumatic brain injury. J. Neuroinflammat. 5:28. doi: 10.1186/1742-2094-5-28

Loane, D. J., and Faden, A. I. (2010). Neuroprotection for traumatic brain injury: translational challenges and emerging therapeutic strategies. Trends Pharmacol. Sci. 31, 596-604. doi: 10.1016/j.tips.2010.09.005

Loane, D. J., Kumar, A., Stoica, B. A., Cabatbat, R., and Faden, A. I. (2014a). Progressive neurodegeneration after experimental brain trauma: association with chronic microglial activation. J. Neuropathol. Exp. Neurol. 73, 14-29. doi: 10.1097/NEN.0000000000000021

Loane, D. J., Stoica, B. A., Tchantchou, F., Kumar, A., Barrett, J. P., Akintola, T., et al. (2014b). Novel mGluR5 positive allosteric modulator improves functional recovery, attenuates neurodegeneration, and alters microglial polarization after experimental traumatic brain injury. Neurotherapeutics 11, 857-869. doi: 10.1007/s13311-014-0298-6

Lockhart, A., Davis, B., Matthews, J. C., Rahmoune, H., Hong, G., Gee, A., et al. (2003). The peripheral benzodiazepine receptor ligand PK11195 binds with high affinity to the acute phase reactant alphal-acid glycoprotein: implications for the use of the ligand as a CNS inflammatory marker. Nucl. Med. Biol. 30, 199-206. doi: 10.1016/S0969-8051(02)00410-9

Mannix, R., Berglass, J., Berkner, J., Moleus, P., Qiu, J., Andrews, N., et al. (2014). Chronic gliosis and behavioral deficits in mice following repetitive mild traumatic brain injury. J. Neurosurg. 121, 1342-1350. doi: 10.3171/2014.7.JNS14272

Maxwell, W. L., MacKinnon, M. A., Stewart, J. E., and Graham, D. I. (2010). Stereology of cerebral cortex after traumatic brain injury matched to the Glasgow outcome score. Brain 133(Pt 1), 139-160. doi: 10.1093/brain/ awp264

McKee, A. C., Alosco, M. L., and Huber, B. R. (2016). Repetitive head impacts and chronic traumatic encephalopathy. Neurosurg. Clin. N. Am. 27, 529-535. doi: 10.1016/j.nec.2016.05.009

McKee, A. C., and Daneshvar, D. H. (2015). The neuropathology of traumatic brain injury. Handb. Clin. Neurol. 127, 45-66. doi: 10.1016/B978-0-444-52892-6.00004-0

McMillan, T. M., Teasdale, G. M., Weir, C. J., and Stewart, E. (2011). Death after head injury: the 13 year outcome of a case control study. J. Neurol. Neurosurg. Psychiatr. 82, 931-935. doi: 10.1136/jnnp.2010.222232

Menzel, L., Kleber, L., Friedrich, C., Hummel, R., Dangel, L., Winter, J., et al. (2017). Progranulin protects against exaggerated axonal injury and astrogliosis following traumatic brain injury. Glia 65, 278-292. doi: 10.1002/glia.23091

Menzies, F. M., Henriquez, F. L., Alexander, J., and Roberts, C. W. (2011). Selective inhibition and augmentation of alternative macrophage activation by progesterone. Immunology 134, 281-291. doi: 10.1111/j.1365-2567.2011.03488.x

Methia, N., Andre, P., Hafezi-Moghadam, A., Economopoulos, M., Thomas, K. L., and Wagner, D. D. (2001). ApoE deficiency compromises the blood brain barrier especially after injury. Mol. Med. 7, 810-815.

Mittelbronn, M., Dietz, K., Schluesener, H. J., and Meyermann, R. (2001). Local distribution of microglia in the normal adult human central nervous system differs by up to one order of magnitude. Acta Neuropathol. 101, 249-255.

Miyazawa, N., Diksic, M., and Yamamoto, Y. (1995). Chronological study of peripheral benzodiazepine binding sites in the rat brain stab wounds using [3H] PK-11195 as a marker for gliosis. Acta Neurochir. (Wien). 137, 207-216. doi: $10.1007 / \mathrm{BF} 02187195$

Morganti, J. M., Jopson, T. D., Liu, S., Riparip, L. K., Guandique, C. K., Gupta, N., et al. (2015). CCR2 antagonism alters brain macrophage polarization and ameliorates cognitive dysfunction induced by traumatic brain injury. $J$. Neurosci. 35, 748-760. doi: 10.1523/JNEUROSCI.2405-14.2015

Morganti, J. M., Riparip, L. K., and Rosi, S. (2016). Call Off the Dog(ma): $\mathrm{M} 1 / \mathrm{M} 2$ polarization is concurrent following traumatic brain injury. PLoS ONE 11:e0148001. doi: 10.1371/journal.pone.0148001

Morohaku, K., Pelton, S. H., Daugherty, D. J., Butler, W. R., Deng, W., and Selvaraj, V. (2014). Translocator protein/peripheral benzodiazepine receptor is not required for steroid hormone biosynthesis. Endocrinology 155, 89-97. doi: 10.1210/en.2013-1556

Murray, P. J., and Wynn, T. A. (2011). Obstacles and opportunities for understanding macrophage polarization. J. Leukoc. Biol. 89, 557-563. doi: $10.1189 / \mathrm{jlb} .0710409$

Nagamoto-Combs, K., McNeal, D. W., Morecraft, R. J., and Combs, C. K. (2007). Prolonged microgliosis in the rhesus monkey central nervous system after traumatic brain injury. J. Neurotrauma 24, 1719-1742. doi: $10.1089 /$ neu. 2007.0377

Nagamoto-Combs, K., Morecraft, R. J., Darling, W. G., and Combs, C. K. (2010). Long-term gliosis and molecular changes in the cervical spinal cord of the rhesus monkey after traumatic brain injury. J. Neurotrauma 27, 565-585. doi: 10.1089/neu.2009.0966

Nishitsuji, K., Hosono, T., Nakamura, T., Bu, G., and Michikawa, M. (2011). Apolipoprotein E regulates the integrity of tight junctions in an isoformdependent manner in an in vitro blood-brain barrier model. J. Biol. Chem. 286, 17536-17542. doi: 10.1074/jbc.M111.225532

O'Connor, W. T., Smyth, A., and Gilchrist, M. D. (2011). Animal models of traumatic brain injury: a critical evaluation. Pharmacol. Ther. 130, 106-113. doi: 10.1016/j.pharmthera.2011.01.001

Ojo, J. O., Mouzon, B., Greenberg, M. B., Bachmeier, C., Mullan, M., and Crawford, F. (2013). Repetitive mild traumatic brain injury augments tau pathology and glial activation in aged hTau mice. J. Neuropathol. Exp. Neurol. 72, 137-151. doi: 10.1097/NEN.0b013e3182814cdf

Omalu, B., Bailes, J., Hamilton, R. L., Kamboh, M. I., Hammers, J., Case, M., et al. (2011). Emerging histomorphologic phenotypes of chronic traumatic encephalopathy in American athletes. Neurosurgery 69, 173-183; discussion 183. doi: $10.1227 /$ neu.0b013e318212bc7b

Owen, D. R., and Matthews, P. M. (2011). Imaging brain microglial activation using positron emission tomography and translocator protein-specific radioligands. Int. Rev. Neurobiol. 101, 19-39. doi: 10.1016/B978-0-12-387718-5.00002-X

Owen, D. R., Yeo, A. J., Gunn, R. N., Song, K., Wadsworth, G., Lewis, A., et al. (2012). An 18-kDa translocator protein (TSPO) polymorphism explains differences in binding affinity of the PET radioligand PBR28. J. Cereb. Blood Flow Metab. 32, 1-5. doi: 10.1038/jcbfm.2011.147

Papadopoulos, V., Baraldi, M., Guilarte, T. R., Knudsen, T. B., Lacapere, J. J., Lindemann, P., et al. (2006). Translocator protein (18kDa): new nomenclature for the peripheral-type benzodiazepine receptor based on its structure and molecular function. Trends Pharmacol. Sci. 27, 402-409. doi: 10.1016/j.tips.2006.06.005

Petraglia, A. L., Plog, B. A., Dayawansa, S., Dashnaw, M. L., Czerniecka, K., Walker, C. T., et al. (2014). The pathophysiology underlying repetitive mild traumatic brain injury in a novel mouse model of chronic traumatic encephalopathy. Surg. Neurol. Int. 5:184. doi: 10.4103/2152-7806.147566

Phelan, H. A., Shafi, S., Parks, J., Maxson, R. T., Ahmad, N., Murphy, J. T., et al. (2007). Use of a pediatric cohort to examine gender and sex 
hormone influences on outcome after trauma. J. Trauma 63, 1127-1131. doi: 10.1097/TA.0b013e318154c1b8

Raghavendra Rao, V. L., Dogan, A., Bowen, K. K., and Dempsey, R. J. (2000). Traumatic brain injury leads to increased expression of peripheral-type benzodiazepine receptors, neuronal death, and activation of astrocytes and microglia in rat thalamus. Exp. Neurol. 161, 102-114. doi: 10.1006/exnr.1999.7269

Ramlackhansingh, A. F., Brooks, D. J., Greenwood, R. J., Bose, S. K., Turkheimer, F. E., Kinnunen, K. M., et al. (2011). Inflammation after trauma: microglial activation and traumatic brain injury. Ann. Neurol. 70, 374-383. doi: 10.1002/ana.22455

Ransohoff, R. M. (2016). A polarizing question: do M1 and M2 microglia exist? Nat. Neurosci. 19, 987-991. doi: 10.1038/nn.4338

Rice, R. A., Spangenberg, E. E., Yamate-Morgan, H., Lee, R. J., Arora, R. P., Hernandez, M. X., et al. (2015). Elimination of microglia improves functional outcomes following extensive neuronal loss in the hippocampus. J. Neurosci. 35, 9977-9989. doi: 10.1523/JNEUROSCI.0336-15.2015

Roberts, G. W., Gentleman, S. M., Lynch, A., Murray, L., Landon, M., and Graham, D. I. (1994). Beta amyloid protein deposition in the brain after severe head injury: implications for the pathogenesis of Alzheimer's disease. J. Neurol. Neurosurg. Psychiatr. 57, 419-425. doi: 10.1136/jnnp.57.4.419

Robinson, S., Berglass, J. B., Denson, J. L., Berkner, J., Anstine, C. V., Winer, J. L., et al. (2017). Microstructural and microglial changes after repetitive mild traumatic brain injury in mice. J. Neurosci. Res. 95, 1025-1035. doi: 10.1002/jnr.23848

Roth, T. L., Nayak, D., Atanasijevic, T., Koretsky, A. P., Latour, L. L., and McGavern, D. B. (2014). Transcranial amelioration of inflammation and cell death after brain injury. Nature 505, 223-228. doi: 10.1038/nature12808

Scott, G., Hellyer, P. J., Ramlackhansingh, A. F., Brooks, D. J., Matthews, P. M., and Sharp, D. J. (2015). Thalamic inflammation after brain trauma is associated with thalamo-cortical white matter damage. J. Neuroinflammat. 12:224. doi: 10.1186/s12974-015-0445-y

Scott, G., Ramlackhansingh, A. F., Edison, P., Hellyer, P., Cole, J., Veronese, M., et al. (2016). Amyloid pathology and axonal injury after brain trauma. Neurology 86, 821-828. doi: 10.1212/WNL.0000000000002413

Selvaraj, V., and Stocco, D. M. (2015). The changing landscape in translocator protein (TSPO) function. Trends Endocrinol. Metab. 26, 341-348. doi: 10.1016/j.tem.2015.02.007

Semple, B. D., Bye, N., Rancan, M., Ziebell, J. M., and Morganti-Kossmann, M. C. (2010). Role of CCL2 (MCP-1) in traumatic brain injury (TBI): evidence from severe TBI patients and CCL2-/- mice. J. Cereb. Blood Flow Metab. 30, 769-782. doi: 10.1038/jcbfm.2009.262

Semple, B. D., Sadjadi, R., Carlson, J., Chen, Y., Xu, D., Ferriero, D. M., et al. (2016). Long-term anesthetic-dependent hypoactivity after repetitive mild traumatic brain injuries in adolescent mice. Dev. Neurosci. 38, 220-238. doi: $10.1159 / 000448089$

Shin, S. S., and Dixon, C. E. (2015). Alterations in cholinergic pathways and therapeutic strategies targeting cholinergic system after Traumatic Brain injury. J. Neurotrauma 32, 1429-1440. doi: 10.1089/neu.2014.3445

Shitaka, Y., Tran, H. T., Bennett, R. E., Sanchez, L., Levy, M. A., Dikranian, K., et al. (2011). Repetitive closed-skull traumatic brain injury in mice causes persistent multifocal axonal injury and microglial reactivity. J. Neuropathol. Exp. Neurol. 70, 551-567. doi: 10.1097/NEN.0b013e31821f891f

Solito, E., and Sastre, M. (2012). Microglia function in Alzheimer's disease. Front. Pharmacol. 3:14. doi: 10.3389/fphar.2012.00014

Soustiel, J. F., Palzur, E., Vlodavsky, E., Veenman, L., and Gavish, M. (2008). The effect of oxygenation level on cerebral post-traumatic apoptotsis is modulated by the $18-\mathrm{kDa}$ translocator protein (also known as peripheral-type benzodiazepine receptor) in a rat model of cortical contusion. Neuropathol. Appl. Neurobiol. 34, 412-423. doi: 10.1111/j.1365-2990.2007.00906.x

Szalay, G., Martinecz, B., Lenart, N., Kornyei, Z., Orsolits, B., Judak, L., et al. (2016). Microglia protect against brain injury and their selective elimination dysregulates neuronal network activity after stroke. Nat. Commun. 7:11499. doi: 10.1038/ncomms11499

Takaki, J., Fujimori, K., Miura, M., Suzuki, T., Sekino, Y., and Sato, K. (2012). L-glutamate released from activated microglia downregulates astrocytic L-glutamate transporter expression in neuroinflammation: the 'collusion' hypothesis for increased extracellular L-glutamate concentration in neuroinflammation. J. Neuroinflammat. 9:275. doi: 10.1186/1742-2094-9-275

Tchantchou, F., and Zhang, Y. (2013). Selective inhibition of alpha/beta-hydrolase domain 6 attenuates neurodegeneration, alleviates blood brain barrier breakdown, and improves functional recovery in a mouse model of traumatic brain injury. J. Neurotrauma 30, 565-579. doi: 10.1089/neu.2012.2647

Thal, S. C., Heinemann, M., Luh, C., Pieter, D., Werner, C., and Engelhard, K. (2011). Pioglitazone reduces secondary brain damage after experimental brain trauma by PPAR-gamma-independent mechanisms. J. Neurotrauma 28, 983-993. doi: 10.1089/neu.2010.1685

Tobinick, E., Kim, N. M., Reyzin, G., Rodriguez-Romanacce, H., and DePuy, V. (2012). Selective TNF inhibition for chronic stroke and traumatic brain injury: an observational study involving 629 consecutive patients treated with perispinal etanercept. CNS Drugs 26, 1051-1070. doi: 10.1007/s40263-012-0013-2

Tran, H. T., LaFerla, F. M., Holtzman, D. M., and Brody, D. L. (2011). Controlled cortical impact traumatic brain injury in $3 \times \mathrm{Tg}-\mathrm{AD}$ mice causes acute intra-axonal amyloid-beta accumulation and independently accelerates the development of tau abnormalities. J. Neurosci. 31, 9513-9525. doi: 10.1523/JNEUROSCI.0858-11.2011

Truettner, J. S., Bramlett, H. M., and Dietrich, W. D. (2016). Posttraumatic therapeutic hypothermia alters microglial and macrophage polarization toward a beneficial phenotype. J. Cereb. Blood Flow Metab. 271678X16680003. doi: $10.1177 / 0271678 \times 16680003$

Tu, L. N., Morohaku, K., Manna, P. R., Pelton, S. H., Butler, W. R., Stocco, D. M., et al. (2014). Peripheral benzodiazepine receptor/translocator protein global knock-out mice are viable with no effects on steroid hormone biosynthesis. $J$. Biol. Chem. 289, 27444-27454. doi: 10.1074/jbc.M114.578286

Turtzo, L. C., Lescher, J., Janes, L., Dean, D. D., Budde, M. D., and Frank, J. A. (2014). Macrophagic and microglial responses after focal traumatic brain injury in the female rat. J. Neuroinflammation 11:82. doi: 10.1186/1742-2094-11-82

Tyburski, A. L., Cheng, L., Assari, S., Darvish, K., and Elliott, M. B. (2017). Frequent mild head injury promotes trigeminal sensitivity concomitant with microglial proliferation, astrocytosis, and increased neuropeptide levels in the trigeminal pain system. J. Headache Pain 18:16. doi: 10.1186/s10194-017-0726-1

Venneti, S., Wagner, A. K., Wang, G., Slagel, S. L., Chen, X., Lopresti, B. J., et al. (2007). The high affinity peripheral benzodiazepine receptor ligand DAA1106 binds specifically to microglia in a rat model of traumatic brain injury: implications for PET imaging. Exp. Neurol. 207, 118-127. doi: 10.1016/j.expneurol.2007.06.003

Verghese, P. B., Castellano, J. M., and Holtzman, D. M. (2011). Apolipoprotein E in Alzheimer's disease and other neurological disorders. Lancet Neurol., 10, 241-252. doi: 10.1016/S1474-4422(10)70325-2

Vile, A. R., and Atkinson, L. (2017). Chronic traumatic encephalopathy: the cellular sequela to repetitive brain injury. J. Clin. Neurosci. 41, 24-29. doi: 10.1016/j.jocn.2017.03.035

Walker, P. A., Bedi, S. S., Shah, S. K., Jimenez, F., Xue, H., Hamilton, J. A., et al. (2012). Intravenous multipotent adult progenitor cell therapy after traumatic brain injury: modulation of the resident microglia population. $J$. Neuroinflammat. 9:228. doi: 10.1186/1742-2094-9-228

Wang, G., Shi, Y., Jiang, X., Leak, R. K., Hu, X., Wu, Y., et al. (2015). HDAC inhibition prevents white matter injury by modulating microglia/macrophage polarization through the GSK3beta/PTEN/Akt axis. Proc. Natl. Acad. Sci. U.S.A. 112, 2853-2858. doi: 10.1073/pnas.1501441112

Wang, G., Zhang, J., Hu, X., Zhang, L., Mao, L., Jiang, X., et al. (2013). Microglia/macrophage polarization dynamics in white matter after traumatic brain injury. J. Cereb. Blood Flow Metab. 33, 1864-1874. doi: $10.1038 /$ jcbfm.2013.146

Wang, Y., Yue, X., Kiesewetter, D. O., Niu, G., Teng, G., and Chen, X. (2014). PET imaging of neuroinflammation in a rat traumatic brain injury model with radiolabeled TSPO ligand DPA-714. Eur. J. Nucl. Med. Mol. Imaging 41, 1440-1449. doi: 10.1007/s00259-014-2727-5

Werner, C., and Engelhard, K. (2007). Pathophysiology of traumatic brain injury. Br. J. Anaesth. 99, 4-9. doi: 10.1093/bja/aem131

Xiong, Y., Mahmood, A., and Chopp, M. (2013). Animal models of traumatic brain injury. Nat. Rev. Neurosci. 14, 128-142. doi: 10.1038/nrn3407 
Xu, L., Nguyen, J. V., Lehar, M., Menon, A., Rha, E., Arena, J., et al. (2016). Repetitive mild traumatic brain injury with impact acceleration in the mouse: multifocal axonopathy, neuroinflammation, and neurodegeneration in the visual system. Exp Neurol 275(Pt 3), 436-449. doi: 10.1016/j.expneurol.2014.11.004

Xue, J., Schmidt, S. V., Sander, J., Draffehn, A., Krebs, W., Quester, I., et al. (2014). Transcriptome-based network analysis reveals a spectrum model of human macrophage activation. Immunity 40, 274-288. doi: 10.1016/j.immuni.2014.01.006

Yu, I., Inaji, M., Maeda, J., Okauchi, T., Nariai, T., Ohno, K., et al. (2010). Glial cell-mediated deterioration and repair of the nervous system after traumatic brain injury in a rat model as assessed by positron emission tomography. J. Neurotrauma 27, 1463-1475. doi: 10.1089/neu.2009.1196

Zanier, E. R., Marchesi, F., Ortolano, F., Perego, C., Arabian, M., Zoerle, T., et al. (2016). Fractalkine receptor deficiency is associated with early protection but late worsening of outcome following Brain Trauma in mice. J. Neurotrauma 33, 1060-1072. doi: 10.1089/neu.2015.4041

Zanier, E. R., Pischiutta, F., Riganti, L., Marchesi, F., Turola, E., Fumagalli, S., et al. (2014). Bone marrow mesenchymal stromal cells drive protective M2 microglia polarization after brain trauma. Neurotherapeutics 11, 679-695. doi: 10.1007/s13311-014-0277-y

Zhang, J., Puvenna, V., and Janigro, D. (2016). "Frontiers in neuroscience: biomarkers of traumatic brain injury and their relationship to pathology," in Translational Research in Traumatic Brain Injury, eds D. Laskowitz and G. Grant (Boca Raton, FL: CRC Press/Taylor and Francis Group), Chapter 12, $1-13$.
Zhang, Z., Zhang, Z. Y., Wu, Y., and Schluesener, H. J. (2012). Immunolocalization of Toll-like receptors 2 and 4 as well as their endogenous ligand, heat shock protein 70, in rat traumatic brain injury. Neuroimmunomodulation 19, 10-19. doi: $10.1159 / 000326771$

Ziebell, J. M., and Morganti-Kossmann, M. C. (2010). Involvement of pro- and anti-inflammatory cytokines and chemokines in the pathophysiology of traumatic brain injury. Neurotherapeutics 7, 22-30. doi: 10.1016/j.nurt.2009.10.016

Ziebell, J. M., Taylor, S. E., Cao, T., Harrison, J. L., and Lifshitz, J. (2012). Rod microglia: elongation, alignment, and coupling to form trains across the somatosensory cortex after experimental diffuse brain injury. J. Neuroinflammat. 9:247. doi: 10.1186/1742-20949-247

Conflict of Interest Statement: The authors declare that the research was conducted in the absence of any commercial or financial relationships that could be construed as a potential conflict of interest. All appropriate permissions have been obtained from the copyright holders of any work that has been reproduced in this manuscript.

Copyright (c) 2017 Donat, Scott, Gentleman and Sastre. This is an open-access article distributed under the terms of the Creative Commons Attribution License (CC BY). The use, distribution or reproduction in other forums is permitted, provided the original author(s) or licensor are credited and that the original publication in this journal is cited, in accordance with accepted academic practice. No use, distribution or reproduction is permitted which does not comply with these terms. 